\title{
Transformations of Electrosensory Encoding Associated with an Adaptive Filter
}

\author{
Nathaniel B. Sawtell and Alan Williams \\ Neurological Sciences Institute, Oregon Health \& Sciences University, Beaverton, Oregon 97006
}

\begin{abstract}
Sensory information is often acquired through active exploration. However, an animal's own movements may result in changes in patterns of sensory input that could interfere with the detection and processing of behaviorally relevant sensory signals. Neural mechanisms for predicting the sensory consequences of movements are thus likely to be of general importance for sensory systems. Such mechanisms have been identified in cerebellum-like structures associated with electrosensory processing in fish. These structures are hypothesized to act as adaptive filters, removing correlations between incoming sensory input and central predictive signals through associative plasticity at parallel fiber synapses. The present study tests the adaptive filter hypothesis in the electrosensory lobe (ELL) of weakly electric mormyrid fish. We compared the ability of electroreceptors and ELL efferent neurons to encode the position of moving objects in the presence and absence of self-generated electrosensory signals caused by tail movements. Tail movements had strong effects on the responses of electroreceptors, substantially reducing the amount of information they conveyed about object position. In contrast, responses of efferent neurons were relatively unaffected by tail movements, and the information they conveyed about object position was preserved. We provide evidence that the electrosensory consequences of tail bending are opposed by proprioceptive inputs conveyed by parallel fibers and that the effects of proprioceptive inputs to efferent cells are plastic. These results support the idea that cerebellum-like structures learn and remove the predictable sensory consequences of behavior and link mechanisms of adaptive filtering to selective encoding of behaviorally relevant sensory information.
\end{abstract}

Key words: synaptic plasticity; cerebellum; electrosensory; neuroethology; proprioception; corollary discharge

\section{Introduction}

Cerebellum-like structures associated with electrosensory systems in fish process information from peripheral electroreceptors in combination with an array of central signals conveyed by parallel fibers (Fig. 1) (Bell, 2002). Parallel fibers convey proprioceptive, motor corollary discharge, and electrosensory signals that could be used to predict the electrosensory consequences of the animals' own behavior. Direct evidence for the generation of such predictions has been obtained from in vivo recordings from cerebellum-like structures in three distinct groups of fish (Bell, 1981; Bastian, 1996; Bodznick et al., 1999). In each case, pairing electrosensory stimuli with central predictive signals results in a change in the neural response to the predictive signals alone that resembles a negative image of the response to the paired stimulus. In vitro studies have demonstrated anti-Hebbian plasticity at parallel fiber synapses onto principal cells that likely underlies negative image formation (Bell et al., 1997b). These results suggest that cerebellum-like circuitry operates as an adaptive filter, generating predictions based on associations between central signals

Received Nov. 2, 2007; revised Dec. 14, 2007; accepted Jan. 4, 2008.

This work was supported by a grant from the National Science Foundation (IOB-0618212) and a National Research Service Award (NS049728-01) to N.B.S. We thank Curtis Bell for helpful discussions and comments on this manuscript.

Correspondence should be addressed to Nathaniel B. Sawtell, Neurological Sciences Institute, Oregon Health \& Sciences University, Beaverton, 0R 97006. E-mail: sawtelln@ohsu.edu.

DOI:10.1523/JNEUROSCI.4946-07.2008

Copyright $\odot 2008$ Society for Neuroscience $\quad$ 0270-6474/08/281598-15\$15.00/0 and sensory inputs and subtracting these predictions from the neural response (Bell, 2001). Adaptive filtering could thus allow external electrosensory signals to be detected and processed more effectively.

Although the circuitry and plasticity mechanisms hypothesized to underlie adaptive filtering have been well studied, the actual transformations of electrosensory encoding associated with such mechanisms have received less attention. The goal of the present study was to provide a quantitative characterization of electrosensory encoding in the presence of self-generated signals caused by movements. For active electrosensory systems, tail movements alter the fish's self-generated electric field and strongly affect activity patterns in electroreceptors (Bastian, 1995; Chen et al., 2005). If the electrosensory lobe (ELL) acts as an adaptive filter, its output neurons should reliably convey information about external sensory stimuli in the presence of such movements.

We tested this hypothesis directly by recording the responses of electroreceptor afferents and ELL efferent cells to small moving conductive and nonconductive objects in the presence and absence of tail movements. We quantified both the relative sensitivity of neural responses to changes in object position versus changes in tail position and the amount of information about object position conveyed by neural responses in the presence and absence of tail movements. Consistent with the adaptive filter hypothesis, ELL efferent cell encoding of object position was far less affected by tail movements than was electroreceptor afferent encoding. 


\section{predictive inputs:}

motor corollary discharge,

other sensory modalities (e.g. proprioception), descending electrosensory
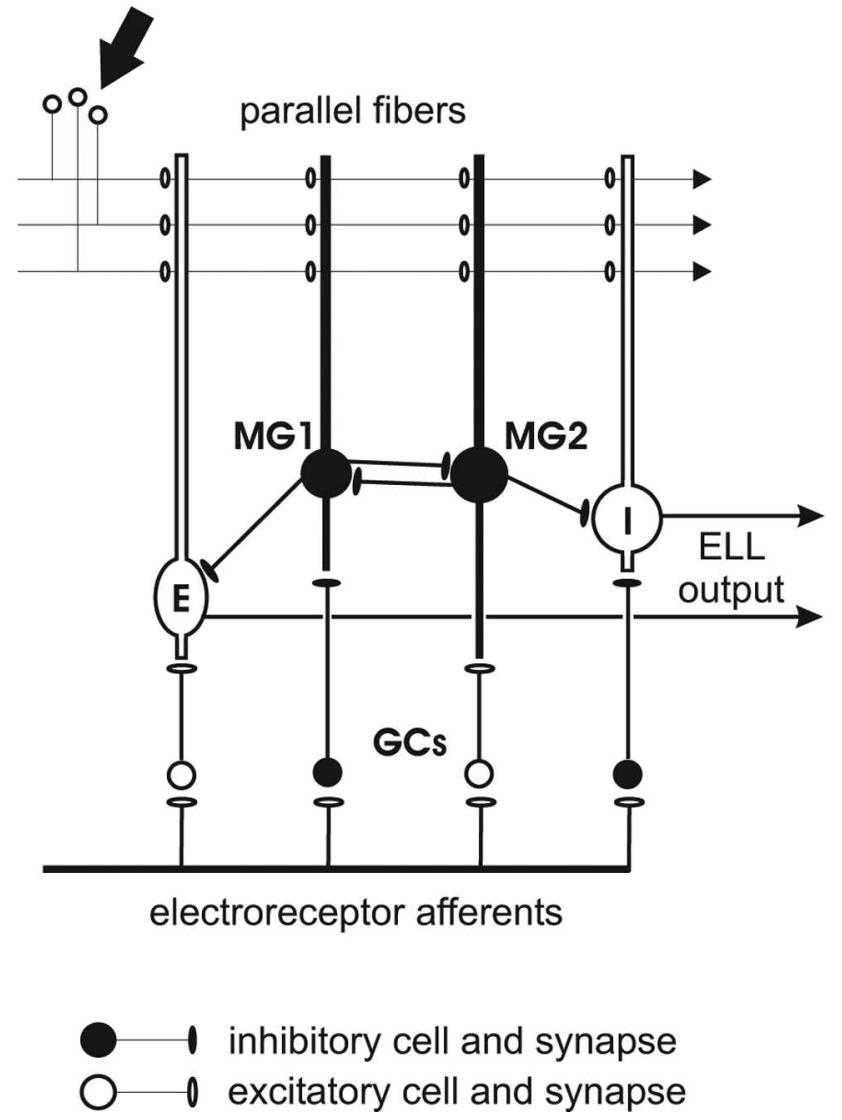

Figure 1. Schematic of the local circuitry of the mormyrid ELL. E- and I-type efferent cells integrate peripheral electrosensory input relayed via granule cells $(G C s)$ with an array of central inputs conveyed by parallel fibers. Purkinje-like MG cells also combine electrosensory and parallel fiber input. I-type MG1 cells inhibit E-type efferent cells, whereas E-type MG2 cells likely inhibit l-type efferent cells. Parallel fiber inputs convey information that could be used to predict changes in incoming electrosensory input.

This transformation appears to be caused, at least in part, by the subtraction of learned associations between central inputs and their sensory consequences. Effects of proprioceptive inputs on efferent cell responses were opposite to the electrosensory effects of tail bending, suggesting that proprioceptive inputs act to oppose the sensory consequences of changes in tail position. We also demonstrate that the effects of proprioceptive inputs on efferent cells are plastic. Pairing an electrosensory stimulus with the electric organ discharge (EOD) motor command at a particular tail position resulted in changes in the response to the EOD motor command that were opposite in sign to the effects of the stimulus and greatest at the paired tailed position.

\section{Materials and Methods}

Experimental preparation. All experiments performed in this study adhere to the American Physiological Society's Guiding Principles in the Care and Use of Animals and were approved by the Institutional Animal Care and Use Committee of Oregon Health \& Sciences University. Mormyrid fish (7-14 cm in length) of the species Gnathonemus petersii were used in these experiments. Fish were anesthetized with metomidate ( $3 \mathrm{mg} / \mathrm{L}$; Aqualife, Vancouver, British Columbia, Canada) and then placed in an experimental chamber $(30 \times 14 \times 9 \mathrm{~cm})$. Fish were held in place using thin, vertically oriented glass rods. Metomidate anesthesia was maintained during the surgery $(1 \mathrm{mg} / \mathrm{L})$ by passing aerated water through the gills ( $\sim 70 \mathrm{ml} / \mathrm{min})$. The rate of the EOD was monitored as an indication of depth of anesthesia. Skin on the surface of the head was removed, and a long-lasting local anesthetic ( $0.75 \%$ bupivacaine) was applied. A plastic rod was secured to the anterior portion of the skull, and a small craniotomy was made overlying the ELL. A well was constructed around the craniotomy using a silicon elastomer (kwik-cast; World Precision Instruments, Sarasota, FL); this allowed the water level in the tank to be raised until the fish was completely submerged. Water conductivity was matched to that of the fish's home tank and maintained close to this value for the entire experiment. Values for water conductivity ranged from 120 to $160 \mu \mathrm{S}$ across experiments.

Anesthesia was removed, and fish were allowed to recover for at least $30 \mathrm{~min}$ before recording. Unanesthetized fish were, for the most part, calm and still with occasional brief bouts of movement. EOD discharge rates were typically $3-5 \mathrm{~Hz}$ when still, with accelerations up to $25 \mathrm{~Hz}$ during movement. If a fish moved frequently or vigorously, the experiment was continued under light metomidate anesthesia $(0.1-0.2 \mathrm{mg} / \mathrm{L})$. Lightly anesthetized fish discharged more slowly and regularly (1.5-3 $\mathrm{Hz}$ ) and moved less frequently. Approximately one-third of our recordings were from lightly anesthetized fish. No differences were observed in neural responses obtained from awake versus lightly anesthetized fish.

Object and tail movements. Stimuli for our experiments were movements of objects and movements of the tail of the fish, both of which altered patterns of EOD-induced current flowing through the skin and activity in electroreceptors. Objects were plastic and metal cylinders moving lateral to the fish, between the tip of the chin appendage or "Schnauzenorgan" and the operculum (Fig. $2 A, E$ ). "Small" objects were $2 \mathrm{~mm}$ in diameter, and "large" objects were $5 \mathrm{~mm}$ in diameter. The cylinders extended from above the water to near the bottom of the tank; thus, the dorsal-ventral position of the object was not a variable in our experiments. Objects were positioned either 5 or $10 \mathrm{~mm}$ away from the fish (measured from the nearest surface of the object to just anterior to the eye). Objects were held by a micromanipulator mounted on a linear stage driven by a servomotor (Pacific Laser Equipment, Santa Ana, CA). The speed of the object movement was $1.5-2 \mathrm{~cm} / \mathrm{s}$.

The fish's tail was held lightly between two glass rods positioned posterior to the electric organ. The rods were held by a manipulator mounted on a second stage. A second pair of rods anterior to the base of the tail prevented the body of the fish from moving. The tail was moved through an arc of approximately $\pm 30^{\circ}$ at a speed of $\sim 30^{\circ} / \mathrm{s}$ (Fig. $2 \mathrm{~A}$ ).

Movement of the stages was driven by analog signals generated by a CED power 1401 (Cambridge Electronics Design, Cambridge, UK) connected to a computer running Spike2 software (Cambridge Electronics Design). For data collection, object and tail were each moved back and forth in a sawtooth pattern. Movements of tail and object were uncorrelated. Pilot experiments also used an irregular sawtooth pattern in which movement from the center position proceeded either to the far position or back to the start position on a random schedule. No differences were observed between neural response to the standard sawtooth and the less predictable stimulus.

Electrophysiology. Local EOD (LEOD) amplitude was recorded using a pair of chlorided silver wires ( $2 \mathrm{~mm}$ spacing) positioned near the skin of the fish, between the eye and the base of the Schnauzenorgan (Fig. 2A,E, red star). LEOD recordings were useful for judging the relative effects of tail and object movements and the size and spatial profiles of field modulations caused by objects. It should be noted, however, that the relative effects of the object were overestimated in the LEOD recordings, because of the fact that the object was significantly closer to the recording dipole than to the skin of the fish.

Extracellular single-unit and field potential recordings were made from the medial zone of ELL using glass pipettes filled with $2 \mathrm{M} \mathrm{NaCl}$ (5-15 $\mathrm{M} \Omega$ ). Higher-resistance pipettes $(30-100 \mathrm{M} \Omega$ ) were used to specifically target electroreceptor afferent fibers. All cells recorded had receptive fields (RFs) on the relatively flat portion of the face near the eye of the fish (Fig. 2 E). A local dipole stimulus delivered $100 \mathrm{~ms}$ after the EOD was used for localizing skin regions that gave rise to neural responses. Intracellular recordings were made using sharp microelectrodes (100$200 \mathrm{M} \Omega$ ) filled with $2 \mathrm{M}$ potassium methylsulfate, as described previously 
(Mohr et al., 2003). Extracellular, intracellular, and field potential recordings were digitally sampled at $20 \mathrm{kHz}$ and LEOD waveforms at 200 $\mathrm{kHz}$ (CED power1401 and Spike2 software; Cambridge Electronics Design).

Identification of cell types. Identification of recorded cell types was aided by previous intracellular recording and labeling studies in which characteristic electric organ corollary discharge (EOCD) and electrosensory responses were linked with cell morphology (Bell et al., 1997a; Mohr et al., 2003). Because ELL is a laminar structure, with different cell types located in different layers, recording location is also useful in identifying cell types. The laminar location of the recording electrode within ELL can be accurately judged based on characteristics of prominent EOD- and EOCD-evoked field potentials (Bell et al., 1992; Engelmann et al., 2006).

Electroreceptor afferents could be unambiguously identified based on recording depth, spike waveforms, and characteristic response patterns (i.e., 1-3 short latency spikes that showed smooth decreases in latency with increases in LEOD amplitude). In many cases, the location of the individual electroreceptor pore innervated by the afferent fiber was identified with a local dipole stimulus.

ELL neurons can be broadly classified as E- or I-cells: E-cells are excited by an increase in LEOD amplitude in the center of their receptive fields, and I-cells are inhibited by such a stimulus. Previous studies have identified several physiologically and morphologically distinct subclasses of E- and I-cells within ELL (Bell et al., 1997a; Mohr et al., 2003). I-cells are located in or just above the ganglion layer and include Purkinje-like interneurons known as type I medium ganglion (MG1) cells, thick smooth dendrite (TSD) cells, and efferent neurons known as large ganglion (LG) cells. All three types of I-cells were encountered in the present study, although we report here mainly on LG cells. TSD cells were recognized by a stereotyped EOCD-evoked burst that was inhibited by an increase in LEOD amplitude. Later spikes in the burst had a lower threshold for inhibition than earlier spikes. MG1 cell recordings were characterized by two distinct spike waveforms, one larger and much less frequent than the other (Sawtell et al., 2007). LG cells were characterized by a low rate of background activity throughout the EOD cycle.

We encountered two classes of E-cells: the first type had no background activity and was located in or just above the ganglion layer; the second type exhibited a low rate of background activity throughout the EOD cycle and was located below the ganglion layer. The latter type almost certainly corresponds to E-type efferent cells known as large fusiform (LF) cells, identified previously using intracellular recording and labeling methods (Bell et al., 1997a; Mohr et al., 2003). The identity of the first type is not known, although responses of these cells are similar to those of morphologically identified type $1 \mathrm{E}$ cells recorded in the preeminential nucleus (Sawtell et al., 2005). The axons of these cells terminate just above the ganglion layer of ELL. With the exception of the analysis of "late" spikes in the latter portion of the Results, responses of these two cell types to tail and object movements were similar and are presented together.

Shunting the EOD. The local EOD was transiently blocked by placing a large metal object near the electric organ. This effectively shunted the current generated by the electric organ and prevented it from flowing dipole.

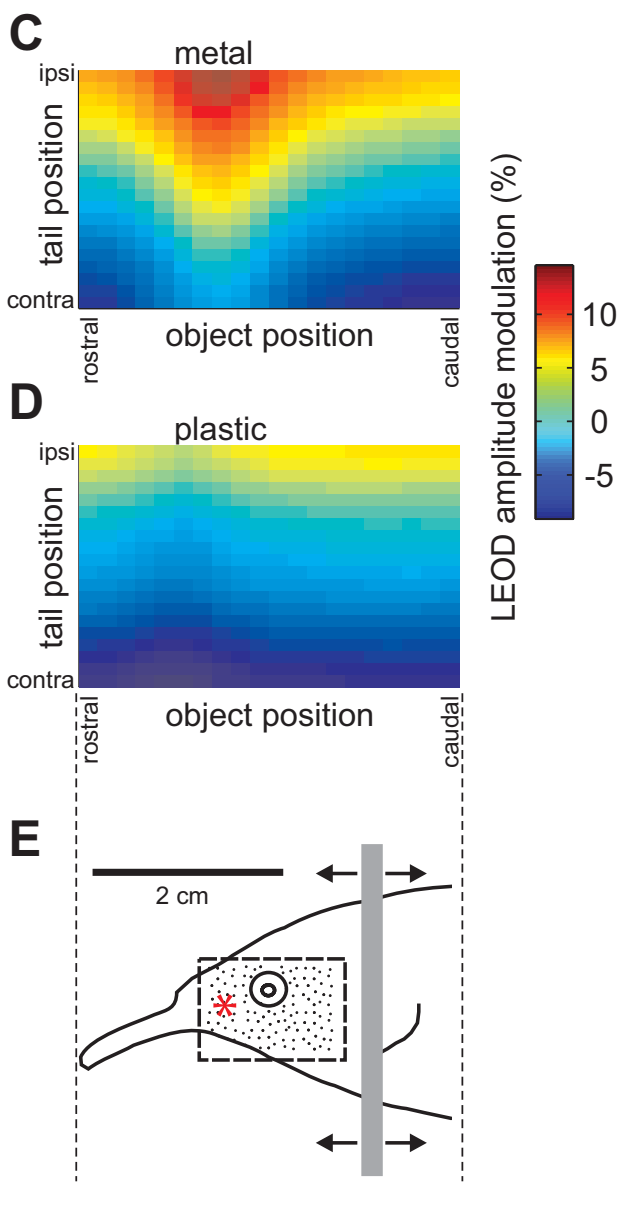

Figure 2. Characterizing electrosensory stimulus encoding in the presence of self-generated signals. $A$, Schematic illustrating the experimental design. Small plastic or metal cylinders were moved alongside the fish (between the tip of the chin appendage

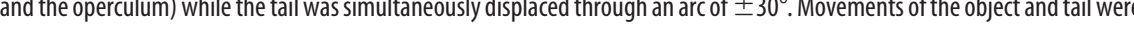
piodic and uncorrelated. Effects of object and tail position on LEOD amplitude were monitored by a recording dipole (red lines) (the fish. $B$, Changes in LEOD amplitude resulting from simultaneous movements of the tail and a $2 \mathrm{~mm}$ waveforms recorded near the skin of the fish at the extremes of the tail movement. Calibration: $40 \mathrm{mV} / \mathrm{cm}, 0.5 \mathrm{~ms}$. C, D, Joint dependence of LEOD amplitude modulation on tail and object position. Data plotted in Care the same as those shown in B. Object in $\boldsymbol{D}$ was a $2 \mathrm{~mm}$ diameter plastic cylinder placed $5 \mathrm{~mm}$ lateral to the fish. $\boldsymbol{E}$, Lateral view of the fish indicating the range of object movement (gray bar) relative to the receptor surface. Scale bar in $\boldsymbol{E}$ applies to $\boldsymbol{C}$ and $\boldsymbol{D}$ as well. Neural recordings were restricted indicate the approximate density of electroreceptors in this area. The red asterisk indicates the position of the LEOD recording

through the rostral portion of the body. The shunt was verified by measuring both the LEOD and EOD-evoked field potential before and after the shunt. The LEOD amplitude near the face was reduced by $>75 \%$. Changes in tail and object position no longer resulted in any modulation of the local field potential recorded in the granular layer, suggesting that the shunt had effectively silenced nearby electroreceptor afferents. This was confirmed directly in several experiments in which electroreceptor afferents were recorded before and after shunting.

Plasticity experiments. For plasticity experiments, gallamine triethiodide (Flaxedil) was given at the end of the surgery $(\sim 20 \mu \mathrm{g} / \mathrm{cm}$ of body length), and the anesthetic was removed. Aerated water was passed over the fish's gills for respiration. Paralysis blocks the effect of electromotoneurons on the electric organ, preventing the EOD, but the motor command signal that would normally elicit an EOD continues to be emitted by the electromotoneurons at a variable rate of 2-5 Hz. Under these conditions, it is possible to examine responses of ELL neurons to central EOCD inputs in isolation from the EOD and to control the electrosensory input that the cells receive. The EOD command signal was 
recorded with a $\mathrm{Ag}-\mathrm{AgCl}$ wire placed over the electric organ. The latencies of EOCD- or command-evoked responses were measured with respect to the negative peak of the first large biphasic wave in the command signal. Plasticity experiments were conducted by moving the tail and recording EOCD-evoked unit responses before, during, and after pairing a local dipole stimulus with the command ( $0.1 \mathrm{~ms}$ square pulse; $10-30$ $\mu \mathrm{A})$. Each pairing lasted $\sim 15 \mathrm{~min}$ and was restricted to a certain range of tail positions (either the middle quarter of the tail range or quarters near the ipsilateral or contralateral extremes of the range). In a majority of cells, pairings were conducted successively at all three positions, with at least $10 \mathrm{~min}$ of recovery between pairings.

Additional plasticity experiments were conducted using intracellular recordings. In these experiments, we paired brief intracellular current injection to evoke spikes $(10-20 \mathrm{~ms} ; 100-400 \mathrm{pA})$ with a certain tail position (extreme ipsilateral, middle, or extreme contralateral). Pairing was performed for 10-15 min. The area of EOCD-evoked synaptic responses was compared across tail positions before and after pairing to assess the significance of plastic changes.

Data analysis. Data analysis was performed in Matlab (MathWorks, Natick, MA). Response maps were constructed from responses to at least 1000 EODs. Tail and object position pairs were binned into a 20-by-20 grid. For each response variable, the response map was defined to be the mean response on this grid, smoothed by a Gaussian filter with an SD of one bin width.

Sensitivity of a response variable to changes in tail or object position was defined to be, for each object and tail position pair, the absolute value of the slope of the response map in the tail or object direction, respectively. Tail-averaged sensitivity curves were obtained by averaging the sensitivity to tail or object, at each object position, across all tail positions. Strength of effect of the tail was quantified by the ratio of the tailaveraged sensitivity to the tail and the tail-averaged sensitivity to the object, at the object position at which the tail-averaged sensitivity to the object was maximal.

For calculating stimulus specific information in a response variable, object position and responses were binned into a 20-by- $n$ grid, with $n=$ 20 for continuous variables (LEOD, latency, and field potential) and $n$ equal to the number of different responses for discrete variables (number of spikes per EOD). For each response bin, response-specific information (RSI) (DeWeese and Meister, 1999; Butts, 2003; Butts and Goldman, 2006) was defined to be the entropy of the object position distribution minus the entropy of the conditional distribution of object position given that response: $\mathrm{RSI}\left(\mathrm{out}_{\mathrm{j}}\right)=H(\mathrm{obj})-H\left(\mathrm{obj} \mid \mathrm{out}_{j}\right)$. This quantity measures the average reduction in uncertainty about object position from observing a particular response. Entropies were estimated via the naive method (maximum likelihood) with Miller-Madow bias correction (Miller, 1955): $H(p)=-\Sigma_{i} p_{i} \log p_{\mathrm{i}}+(m-1) / 2 N$, where $m$ is the number of nonzero $p_{i}$, and $N$ is the number of data points. Response bins with $<20$ responses were excluded. For each object position, stimulusspecific information (SSI) (Butts, 2003; Butts and Goldman, 2006) was defined to be the mean RSI over all responses occurring at that object position, weighted by their probability of occurrence: $\operatorname{SSI}\left(\mathrm{obj}_{i}\right)=$ $\Sigma_{j} p\left(\right.$ out $\left._{j} \mid \mathrm{obj}_{i}\right) \mathrm{RSI}\left(\right.$ out $\left._{j}\right)$. This quantity measures the average reduction in uncertainty about object position (within an ensemble) from observing the responses elicited by a particular object position. SSI for object position was computed under conditions in which both tail and object positions varied $\left(\mathrm{SSI}_{\mathrm{both}}\right)$ and in which only object position varied $\left(\mathrm{SSI}_{\mathrm{obj}}\right)$. Strength of effect of the tail was quantified by $\left(\mathrm{SSI}_{\mathrm{obj}}-\mathrm{SSI}_{\text {both }}\right) / \mathrm{SSI}_{\mathrm{obj}}$, the relative reduction in object SSI when tail position varied, at the object position at which SSI $_{\text {obj }}$ was maximal.

Late spike analysis. "Early" spikes were defined to be those occurring $<100 \mathrm{~ms}$ after the preceding EOD, and "late" spikes were defined as those occurring at least $400 \mathrm{~ms}$ after the EOD. For both early and late spikes, histograms of spike count versus tail position at EOD time $\left(\right.$ tail $\left._{\mathrm{EOD}}\right)$ and versus tail position at spike time ( tail $_{\mathrm{SP}}$ ) were computed. Because the tail movement protocols used here were periodic, tail $\mathrm{SP}_{\mathrm{SP}}$ and tail $_{\mathrm{EOD}}$ were (for spikes with a given latency) strongly correlated. To assess whether spike occurrence was more strongly tied to tail position at EOD time or tail position at spike time, we randomly reassigned tail ${ }_{\mathrm{EOD}}$ or tail $l_{\mathrm{SP}}$ according to their conditional distribution given tail $l_{\mathrm{SP}}$ or tail $_{\mathrm{EOD}}$, respectively, and recomputed the spike count histograms. If spike occurrence was influenced directly by tail $\mathrm{EOD}_{\text {D }}$ but only indirectly by tail $_{\mathrm{SP}}$ (via its correlations with tail ${ }_{\mathrm{EOD}}$ ), then reassigning tail $\mathrm{SP}_{\mathrm{S}}$ in this way should have no effect on the histogram of spike count versus tail $1_{\mathrm{SP}}$. Conversely, if spikes depended directly on tail ${ }_{\mathrm{SP}}$ but only indirectly on tail $_{\mathrm{EOD}}$, then reassigning tail $\mathrm{EOD}_{\mathrm{EO}}$ should have no effect on the histogram versus tail $_{\mathrm{EOD}}$.

\section{Results}

Weakly electric fish use active electrolocation to navigate, avoid predators, and find prey in darkness. These fish possess specialized electric organs in their tails that emit weak electrical fields, known as EODs, and specialized electroreceptors distributed over the body surface that respond to changes in these fields. As fish move through their environment, spatial patterns of EOD amplitude across the receptor surface will change both as a result of nearby objects with conductivity different from the surrounding water and as a result of the movements of the fish itself. Changes in the position of electroreceptors relative to the electric organ resulting from movements of the tail cause changes in EOD amplitude that may be substantially larger than those caused by many behaviorally relevant external stimuli (Bastian, 1995; Chen et al., 2005). The initial goal of the present study was to characterize encoding of naturalistic electrosensory stimuli in the presence of self-generated signals caused by tail movements.

We recorded extracellular single-unit activity in the medial zone of ELL, while simultaneously bending the tail and moving small objects (plastic or metal cylinders) alongside an awake or lightly anesthetized fish (Fig. $2 A, E$ ). Changes in electrosensory reafference caused by tail and object movements were assessed by measuring the LEOD amplitude near the skin of the fish at one position along the path of the object (Fig. $2 \mathrm{~A}$, red lines). Ipsilateral tail bends (i.e., toward the recording dipole) increased local LEOD amplitude, whereas contralateral bends decreased LEOD amplitude. Modulations were approximately linear as a function of tail position and ranged from \pm 5 to $15 \%$ (Fig. 2 B, top). Effects of objects depended on the object material, size, and distance from the skin. Metal objects increased LEOD amplitude (Fig. $2 C$ ), whereas plastic objects decreased LEOD amplitude (Fig. $2 D)$. The contrast in conductivity between the object and the surrounding water was greater for metal than for plastic objects; thus, metal objects had substantially larger effects on LEOD amplitude than plastic objects of the same size (Fig. 2, compare $C$, D).

All recorded units had receptive fields on the relatively flat portion of the face around the eye (Fig. 2 E). Electroreceptor afferents as well as E- and I-type ELL efferent cells could be identified based on recording depth and characteristic response patterns (see Material and Methods). After recording responses of an E- or I-cell to movements of the tail and object, we often recorded granular layer field potential (GFP) responses to the same stimuli. Changes in the peak amplitude of the early negative component of the GFP reflect electroreceptor afferent-evoked synaptic activity and were used as a convenient proxy for responses of electroreceptor afferents (Gomez et al., 2004; Engelmann et al., 2006). Recordings of the GFP allowed us to compare responses across stages on the same electrode track in response to the same stimuli, thus minimizing variability caused by differences across recordings and across fish in the relative strength of the effects of object versus tail movements.

\section{Transformations of electrosensory encoding}

Responses of a representative electroreceptor afferent, E-cell, and I-cell to simultaneous object and tail movements are illustrated in 


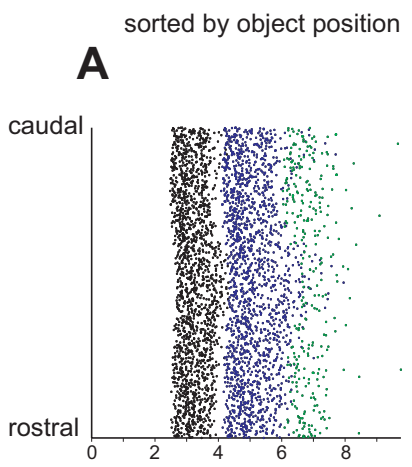

C

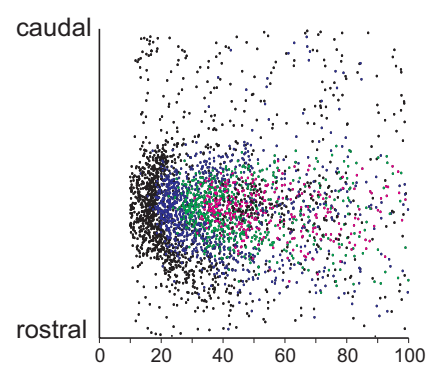

E

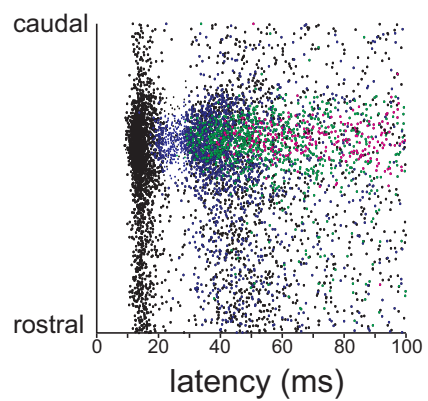

\section{B}
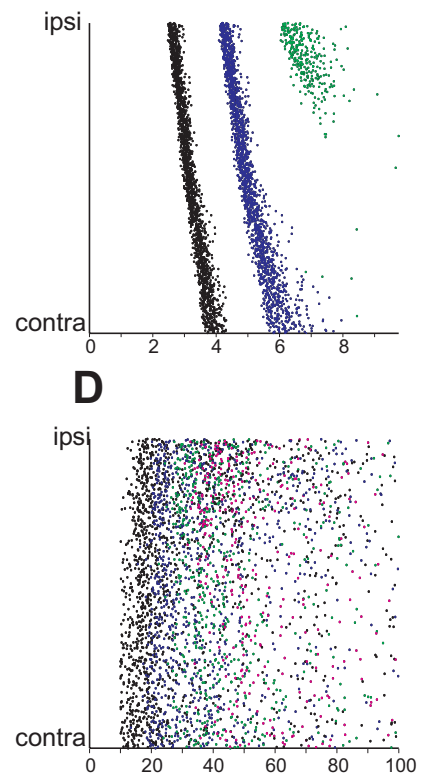

$\mathbf{F}$

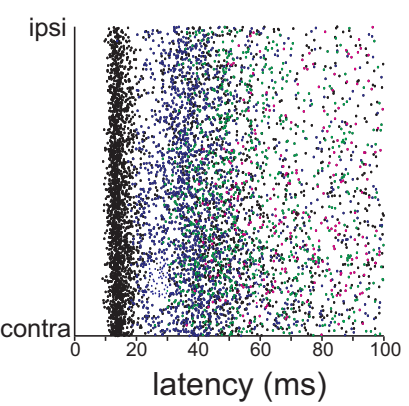

Figure 3. Effects of simultaneous movement of object and tail on neural responses across processing stages. Rasters in the left column are sorted by position of the object alone and rasters in the right column by the position of the tail alone. Colors indicate the first (black), second (blue), third (green), and subsequent (pink) spikes that occurred after the EOD. $\boldsymbol{A}, \boldsymbol{B}$, Responses of an electroreceptor afferent to simultaneous movements of the tail and a $2 \mathrm{~mm}$ diameter plastic cylinder placed $5 \mathrm{~mm}$ lateral to the fish. Note the strong dependence on tail position in $\boldsymbol{B}$ and the much weaker dependence on object position in $\boldsymbol{A}$. $\boldsymbol{C}, \boldsymbol{D}$, Responses of an E-cell to simultaneous movements of the tail and a $2 \mathrm{~mm}$ diameter metal cylinder placed $5 \mathrm{~mm}$ lateral to the fish. Note a clear dependence of the response on object position and a much weaker dependence on tail position. $\boldsymbol{E}, \boldsymbol{F}$, Responses of an I-cell to simultaneous movements of the tail and a $2 \mathrm{~mm}$ diameter plastic cylinder placed $5 \mathrm{~mm}$ lateral to the fish. Again, note a clear dependence of the response on object position and a much weaker dependence on tail position.

Figure 3. Responses are displayed as rasters sorted either by the position of the object alone (Fig. 3, left column) or by the position of the tail alone (Fig. 3, right column). A-type electroreceptor afferents fire 1-4 precisely timed spikes after each EOD, responding to an increases in EOD amplitude with a decrease in spike latency, and for sufficiently large changes in EOD amplitude, an increase in spike number (Bell, 1990b; Sawtell et al., 2006).

For the electroreceptor afferent shown in Figure 3, a plastic object caused an increase in spike latency when the object was near the receptor pore (Fig. 3A). However, effects of object position on latency were quite small compared with the variance of the response at a given object position. Most of this variance was attributable to the position of the tail (Fig. 3B), with an ipsilateral tail bend causing a decrease in spike latency as well as an increase in spike number. These responses are consistent with the relative

effects of tail and object position on LEOD amplitude (Fig. 2D) and suggest that electroreceptor afferent encoding of object position is strongly affected by the position of the tail.

E- and I-type efferent cells of the mormyrid ELL combine input from electroreceptor afferents (relayed via granular cells) with massive central inputs (relayed via parallel fibers and Purkinje-like interneurons) (Fig. 1) (Meek et al., 1999). E-cells were excited by metal objects (Fig. 3C) and I-cells by plastic objects (Fig. $3 E$ ), both responding with increases in spike number and, in many cases, with graded decreases in spike latency. These responses were substantially slower and less temporally precise than those observed in electroreceptor afferents. In contrast to electroreceptor afferents, E- and I-cells responded more strongly to movements of objects than to movements of the tail (Fig. $3 D, F)$.

The full joint dependence of these responses on tail and object position is illustrated in Figure 4. Warm colors indicate excitatory responses: decreases in spike latency for electroreceptor afferents, increases in amplitude for GFP recordings, and increases in spike number for E- and I-cells. The dependence of electroreceptor afferent spike latency on tail and object position and object material is similar to that of LEOD amplitude (compare Figs. $4 A, B$, $2 C, D)$. The width and asymmetrical profile of the dependence of electroreceptor afferent spike latency on object position are consistent with characteristics of electrical images described in previous experimental and modeling studies (Gomez et al., 2004; Migliaro et al., 2005). Again it is evident that responses of electroreceptor afferents to an object at a given location depend strongly on the position of the tail.

In contrast, response maps for two representative E-cells (Fig. $4 C, D$, top) and two representative I-cells (Fig. $4 E, F$, top) reveal responses to object position that are much less affected by, and in some cases nearly invariant to, changes in tail position. GFP (Fig. $4 D-F$, bottom) and electroreceptor afferent (Fig. 4C, bottom) recordings made on the same electrode track show strong effects of tail position, suggesting a genuine transformation across processing stages.

Changes in the spatial structure of electrosensory receptive fields are also evident. E- and I-cells displayed narrower and sharper receptive fields than individual electroreceptor afferents, despite receiving convergent afferent input. Intracellular recording studies have provided evidence for inhibitory centersurround receptive field organization in ELL efferent cells (Bell and Grant, 1992; Bell et al., 1997a), and such mechanisms likely contribute to the apparent sharpening of E- and I-cell receptive fields observed here. Spatial filtering, resulting from centersurround receptive field structures, could also contribute to the minimal effects of tail bending on changes caused by small, nearby objects that we observed in E- and I-cells, because changes in LEOD amplitude caused by tail bends would be large and spatially uniform.

It is also important to note that although they were reduced, effects of tail position on E- and I-cell responses were not entirely absent. Note the clear gradients in the peak magnitude of the object response as tail position varies, for the E-cell shown in Figure $4 C$ and the I-cell shown in Figure $4 E$. In both cases, the effects of an ipsilateral tail bend are in the expected direction, increasing response magnitude for the E-cell and decreasing response magnitude for the I-cell.

We took two approaches to quantifying the relative effects of tail and object position across cells and stages. The first was to compare the relative sensitivity of neural responses to changes in object versus tail position (see Material and Methods). Figure 
A
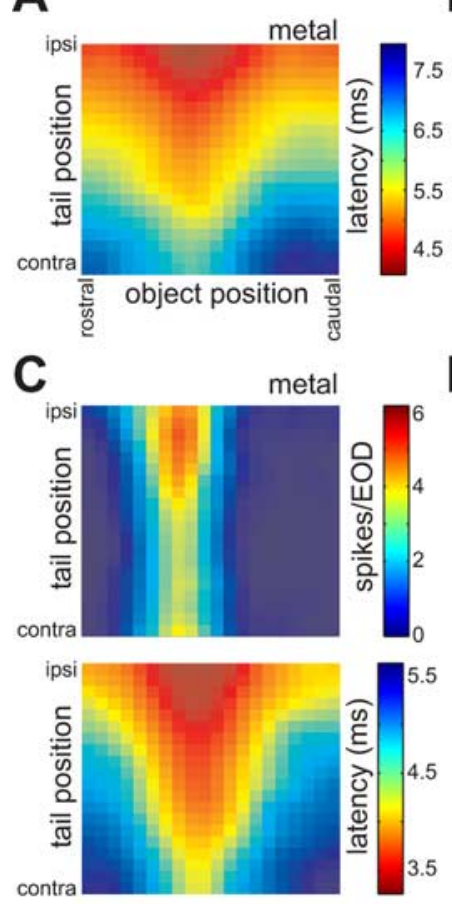

E
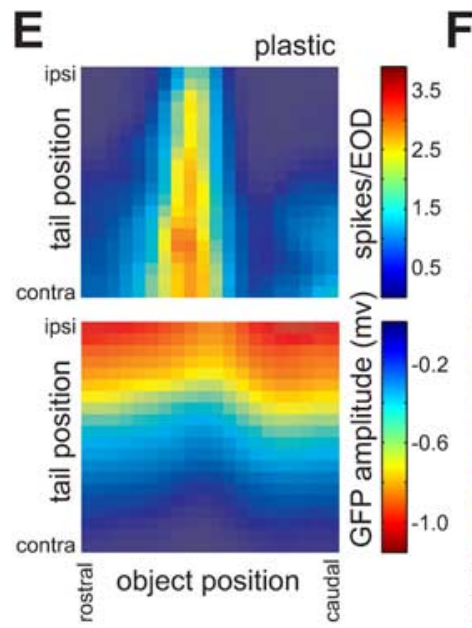

$\mathbf{F}$
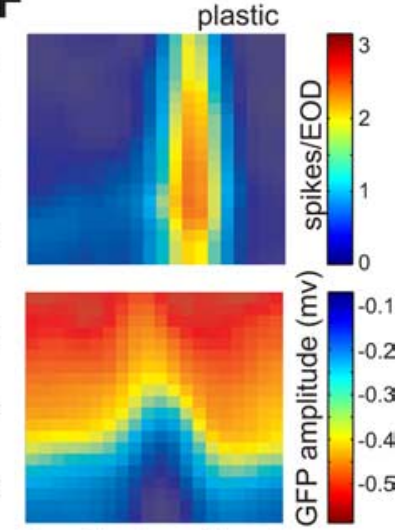

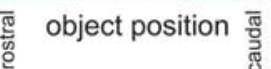

Figure 4. Response maps illustrating the joint dependence of neural responses on object and tail position across processing stages. $\boldsymbol{A}, \boldsymbol{B}$, Dependence of electroreceptor afferent firstspike latency on tail and object position, for two afferents. Objects were $2 \mathrm{~mm}$ diameter metal $(\boldsymbol{A})$ and plastic $(\boldsymbol{B})$ cylinders placed $5 \mathrm{~mm}$ lateral to the fish. Data from $\boldsymbol{B}$ are the same as those plotted in Figure 3, $A$ and $B$. C, D, Top, Dependence of spike number (i.e., the number of spikes within $100 \mathrm{~ms}$ after the EOD) on tail and object position for two E-cells. For both units, the object was a $2 \mathrm{~mm}$ diameter metal cylinder placed $5 \mathrm{~mm}$ lateral to the fish. Bottom, Responses of an electroreceptor afferent $(\boldsymbol{C})$ and the granular field potential $(\boldsymbol{D})$ recorded on the same electrode track and in response to stimuli identical to those used for collecting the data shown in the maps above. Data in the top panel in $\boldsymbol{C}$ are the same as those plotted in Figure 3, C and D.E, $\boldsymbol{F}$, Top, Dependence of spike number on tail and object position for two I-cells. For both units, the object was a $2 \mathrm{~mm}$ diameter plastic cylinder placed $5 \mathrm{~mm}$ lateral to the fish. Bottom, Granular field potential responses recorded on the same electrode track and in response to stimuli identical to those used for collecting the data shown in the maps above. Data in the top panel in $\boldsymbol{F}$ are the same as those plotted in Figure 3, $E$ and $F$.

$5 B-F$ shows tail-averaged sensitivity curves as a function of object position. These were obtained by averaging the sensitivity to changes in tail or object position, at each object position, across all tail positions. As is evident in the examples shown in Figure 5, electroreceptor afferents (Fig. $5 A, B$ ) were relatively more sensitive to changes in tail position than were E- and I-cells (Fig.
A

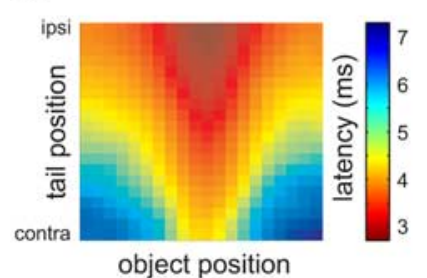

C

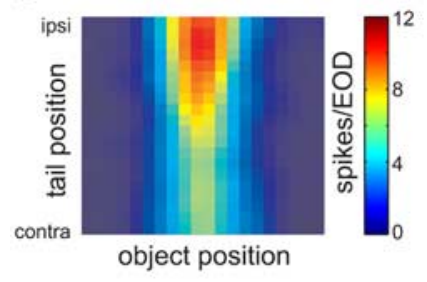

E

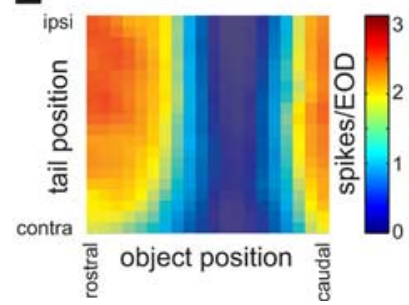

B

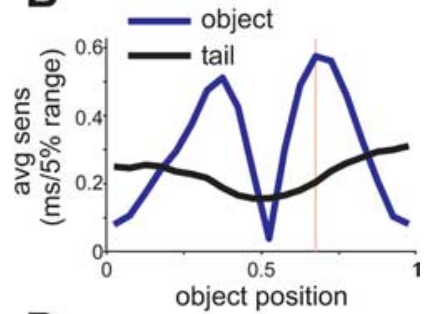

D

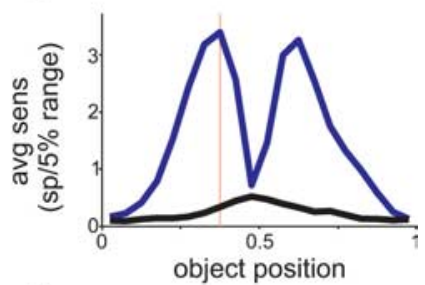

$\mathbf{F}$

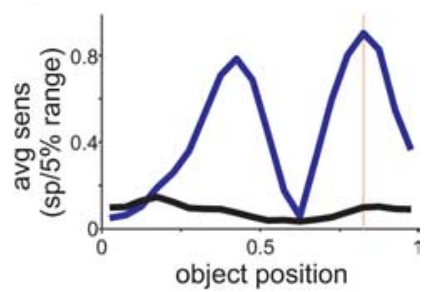

Figure 5. Relative sensitivity of responses to object and tail position across processing stages. $\boldsymbol{A}$, Response map for an electroreceptor afferent illustrating the dependence of firstspike latency on tail and object position. The object was a $2 \mathrm{~mm}$ diameter metal cylinder placed $5 \mathrm{~mm}$ lateral to the fish. $\boldsymbol{B}$, Tail-averaged sensitivity to changes in object position (blue line) and tail position (black line), as a function of object position, calculated from the responses in $\boldsymbol{A}$. Ordinate values represent the change in neural response for a change in object position of $5 \%$ of its total range (the distance from the tip of the chin appendage to the operculum). Note that sensitivity to changes in object position is greatest at the edges of the receptive fields. The object position at which sensitivity was greatest is indicated by the red line. 0 bject position: $0=$ rostral; 1 = caudal. $\boldsymbol{C}, \boldsymbol{E}$, Response maps for E-cell and I-cells illustrating the dependence of spike number on tail and object position. The object was a $2 \mathrm{~mm}$ diameter metal cylinder placed $5 \mathrm{~mm}$ lateral to the fish. The E-cell in C was excited when the metal object passed through the center of its RF, whereas the I-cell in $\boldsymbol{E}$ was inhibited. $\boldsymbol{D}, \boldsymbol{F}$, Tail-averaged sensitivity to changes in object and tail position as a function of the position of the object calculated from the responses in C. Note that the relative sensitivity to changes in object versus tail position is greater for the E- and I-cells than for the electroreceptor afferent shown above.

$5 C-F)$. The I-cell in Figure 5, $E$ and $F$, is strongly inhibited by a metal object but virtually unaffected by tail position.

As a single measure of the relative effect of tail versus object position, we defined the sensitivity ratio for each response map, as the ratio of the tail-averaged sensitivity to the tail and the tail-averaged sensitivity to the object, at the object position at which the tail-averaged sensitivity to the object was greatest (typically at the edge of the receptive field) (Fig. $5 B, D, F$, red lines). Sensitivity ratios for electroreceptor afferents, E- and I-cells, and GFP recordings are compared in Figure 6A. Overall, sensitivity ratios were higher in electroreceptor afferents and GFPs than in E- and I-cells, consistent with the hypothesis that effects of selfgenerated signals are reduced across processing stages. As would be expected, sensitivity ratios for electroreceptor afferents and GFPs depended on properties of the object: objects with stronger effects on the LEOD were associated with smaller sensitivity ratios. This trend was small or absent in E- and I-cells. For $26 \mathrm{E}-$ and I-cells, we recorded GFP or electroreceptor afferent responses on the same electrode track using the same stimuli. In all cases, the 
sensitivity ratio was smaller for the E- or I-cells, again consistent with a genuine transformation across processing stages (Fig. 6B).

In a subset of units, we also recorded responses to object movements in the absence of tail movements. We then used information theory to ask how much information about object position was contained in the neural response in the presence and absence of tail movements (see Materials and Methods). We expected that tail movements should be associated with a substantial loss of information in electroreceptor afferents. On the other hand, if effects of tail position have been effectively removed in E- and I-cells, then information about object position should be similar in the presence and absence of tail movements.

This analysis also provided a framework for making functional comparisons across stages and cell types and for comparing the amount of information conveyed by different candidate neural codes (i.e., first-spike latency versus spike number). Figure 7 illustrates this analysis applied to a representative electroreceptor afferent (same as in Fig. 5A) and a representative E-cell (same as in Fig. 5C).

Effects of object position on electroreceptor afferent spike latency in the presence and absence of tail movements are shown in Figure $7 A$. Note that for a given spike latency, uncertainty about object position is substantially greater when tail position varies (Fig. $7 A_{1}, A_{2}$ ). We quantified this observation by comparing the RSI and the SSI in the presence and absence of tail movements (DeWeese and Meister, 1999; Butts, 2003; Butts and Goldman, 2006). The RSI is a measure of the information about a stimulus associated with a given neural response. For the electroreceptor afferent shown in Figure $7 A$, the most informative responses are the shortest latency spikes that occurred when the object was nearest the receptor pore (Fig. $7 A_{3}$ ). For these experiments, one bit of information about object position means, roughly, that the object can be localized to one-half of its total range; two bits that it can be localized to one-fourth of its range; and so on.

The SSI is a measure of the information associated with a given stimulus: the average of the RSI of all of the responses associated with a given object position weighted by the frequency of their occurrence. For the same electroreceptor afferent, the peak SSI occurs, as expected based on the RSI, for object positions associated with the shortest latencies (i.e., those nearest the receptor pore) (Fig. $7 A_{4}$ ). It is clear from both the RSI and SSI that tail movements result in a significant loss of information about object position conveyed by electroreceptor afferent spike latency (Fig. $7 A_{3}, A_{4}$, compare gray and black curves).

Figure $7 B$ shows the results of the same analysis for the same electroreceptor afferent, but considering spike number instead of spike latency as the coding variable. As was the case for latency, tail movements resulted in a substantial loss of information about object position conveyed by changes in spike number (Fig. $7 B_{3}, B_{4}$, compare gray and black curves). We also found that, for electroreceptor afferents, spike latency carries substantially more information about object position than spike number (peak SSI in the absence of tail movements: $1.3 \pm 0.3$ bits for latency vs $0.4 \pm 0.3$ for spike number; $p<0.01$, paired $t$ test; $n=7$ ).
B

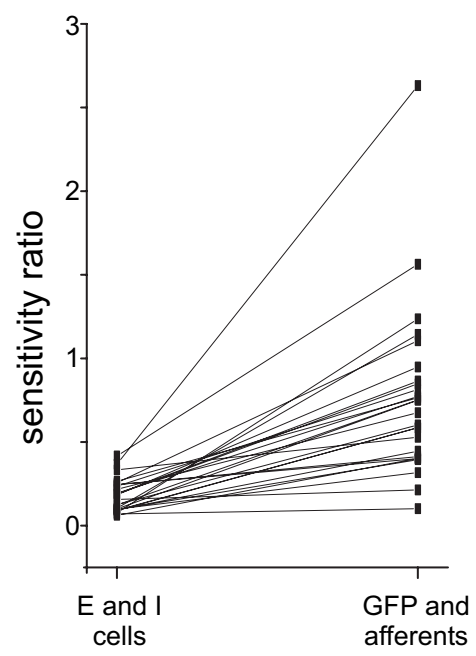

Figure 6. Summary of the sensitivity analysis for different object materials, sizes, and distances. $\boldsymbol{A}$, Sensitivity ratios for all electroreceptor afferents, GFP recordings, E- and I-cells grouped by stimulus condition (see legend). Means for each group are indicated by the height of the bars and individual experiments by horizontal lines. A smaller sensitivity ratio indicates a weaker effect of tail position. $\boldsymbol{B}$, Sensitivity ratios for $\mathrm{E}$ - and $\mathrm{I}$-cells and for electroreceptor afferent or GFP recordings made on the same electrode track under identical stimulus conditions. In all cases, the sensitivity ratio was smaller for the E- or I-cell.

Little quantitative information is available regarding how ELL neurons in pulse-type electric fish encode electrosensory stimuli. For the representative E-cell shown in Figure 7, it can be seen that changes in object position were associated with both decreases in first-spike latency (Fig. $7 C_{1}$ ) and increases in spike number (Fig. $7 D_{1}$ ). Latency shifts in E- and I-cells occurred on a much coarser time scale than the latency shifts observed in electroreceptor afferents (Fig. 7, compare $A_{1}, C_{1}$ ). Moreover, clear first-spike latency shifts were evident in only a subset of recorded E- and I-cells, whereas all E- and I-cells showed clear changes in spike number. The reverse was true for electroreceptor afferents, i.e., all afferents showed a clear shift in latency, but only a subset were driven strongly enough by our stimuli to alter spike number. For the subset of E- and I-cells that did exhibit clear latency shifts, we found that the amount of information about object position conveyed by latency changes was slightly less than that conveyed by spike number (peak SSI in the absence of tail movements: $1.3 \pm$ 0.2 bits for latency vs $1.5 \pm 0.2$ for spike number; $p<0.05$, paired $t$ test; $n=11)$. It is also interesting to note that, under the conditions of our experiments, changes in spike number in individual E- and I-cells conveyed approximately the same amount of information as changes in spike latency in individual electroreceptor afferents [peak SSI in the absence of tail movements: $1.4 \pm 0.7$ bits for E- and I-cells $(n=23)$ vs $1.3 \pm 0.3$ bits for electroreceptor afferents $(n=7)]$.

Consistent with the adaptive filter hypothesis, responses of many E- and I-cells conveyed virtually the same amount of information about object position in the presence and absence of tail movements (Fig. 7D). It is interesting to note that cells whose responses varied noticeably with tail position could nevertheless provide unambiguous information about object position (compare response map in Fig. $5 C$ with SSI curves for the same unit shown in Fig. 7D). It was also clear in some E- and I-cells that the amount of information loss associated with tail movements depended on the choice of coding variable (Fig. 7, compare $C_{4}, D_{4}$ ).

To compare the amount of information lost because of tail movements across stages, we plotted the peak SSI in the presence 

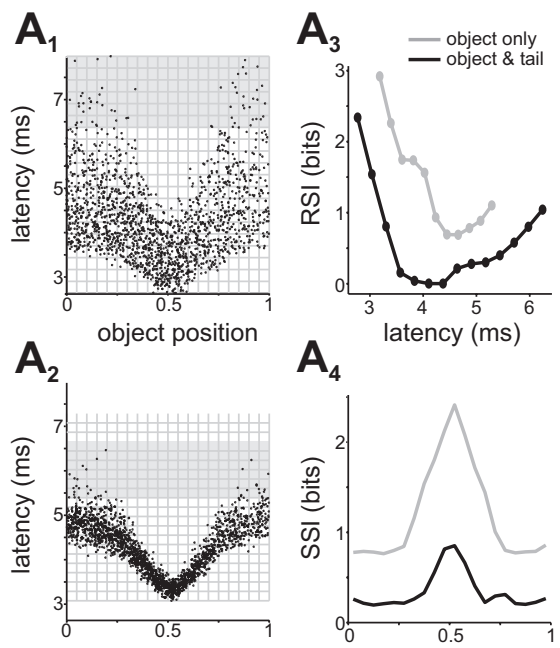

object position

$\mathrm{C}_{1}$

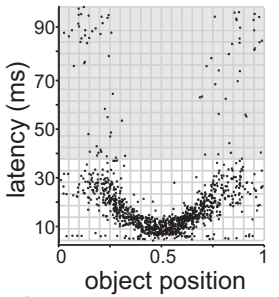

$\mathrm{C}_{2}$

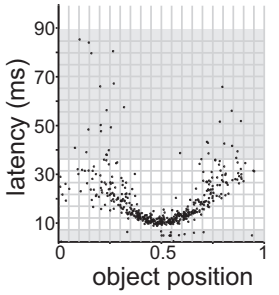

$\mathrm{C}_{3}$

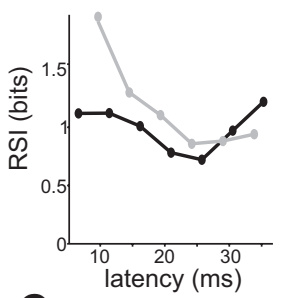

$\mathrm{C}_{4}$

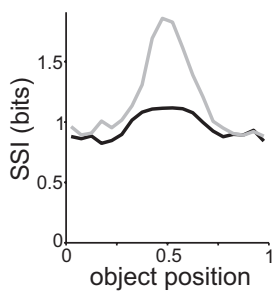

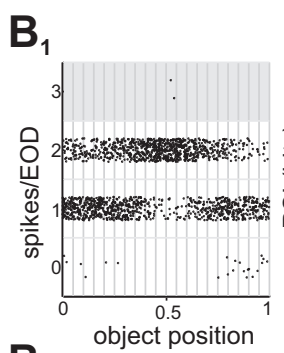

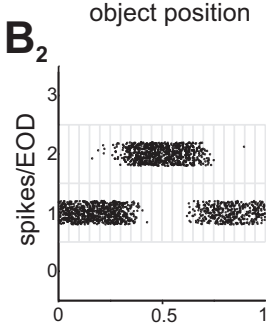

object position

$\mathrm{B}_{3}$
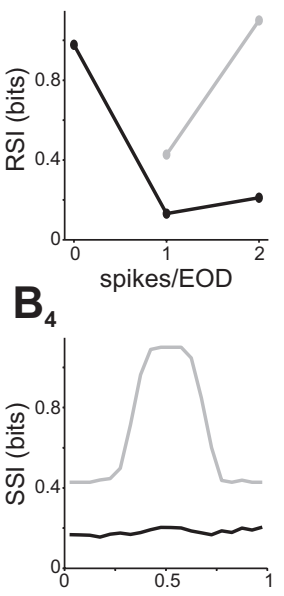

object position

$\mathbf{D}_{1}$
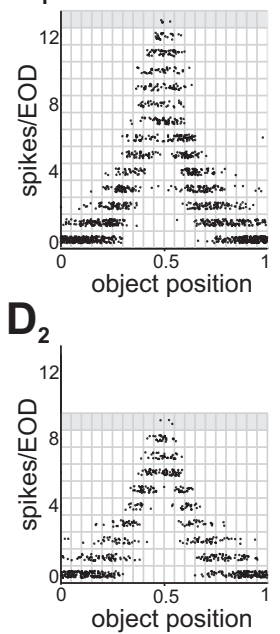

$\mathbf{D}_{3}$

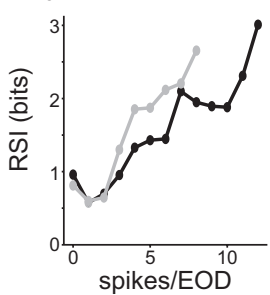

$\mathrm{D}_{4}$

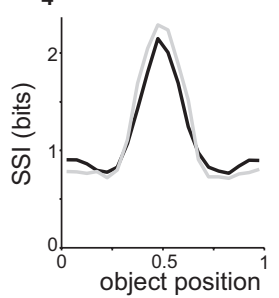

Figure 7. Information about object position conveyed by neural responses across processing stages, in presence or absence of tail movements. $\boldsymbol{A}_{\boldsymbol{1}}$, Scatter plot of first-spike latencies versus object position for an electroreceptor afferent, in the presence of tail movement. The object was a $2 \mathrm{~mm}$ diameter metal cylinder placed $5 \mathrm{~mm}$ lateral to the fish. Data are the same as those shown in the color map in Figure $5 \mathrm{~A}$. The grid indicates response and object bins used for information calculations (see Materials and Methods). Gray regions indicate undersampled responses excluded from subsequent analysis. 0 bject position: $0=$ rostral; $1=$ caudal. $\boldsymbol{A}_{2}$, Responses of the same electroreceptor afferent in the absence of tail movement. Note the reduced variability in the response. $\boldsymbol{A}_{3}$, RSI calculated from the data shown in $\boldsymbol{A}_{1}$ (black) and $\boldsymbol{A}_{2}$ (gray). $\boldsymbol{A}_{4}$, SSI calculated from the data shown in $\boldsymbol{A}_{1}$ (black) and $\boldsymbol{A}_{\mathbf{2}}$ (gray). Note that tail movements (black) cause substantial reductions in RSI and SSI. $\boldsymbol{B}_{\mathbf{1 - 4}}$, Same data and analysis as in $\boldsymbol{A}_{\mathbf{1 - 4}}$ but with spike number as the output variable. Note that changes in spike number convey substantially less information about object position than changes in first-spike latency. $\boldsymbol{C}_{1-4}$, Same analysis for first-spike latency in an E-cell. The object was a $2 \mathrm{~mm}$ diameter metal cylinder placed $5 \mathrm{~mm}$ lateral to the fish. Data in $\boldsymbol{C}_{1}$ are the same as those shown in the color map in Figure 5C. $\boldsymbol{D}_{1-4}$, Same data and analysis as in $\boldsymbol{C}_{\mathbf{1 - 4}}$ but with spike number as the output variable. Note that the RSI and SSI are virtually unaffected by tail movements.

of tail movements versus the peak SSI in the absence of tail movements for GFPs, electroreceptor afferents (using latency as the coding variable), and E- and I-cells (using spike number as the coding variable) (Fig. $8 \mathrm{~A}$ ). Although the amount of information conveyed by individual units at different processing stages was similar in the absence of tail movements, in the presence of tail movements E- and I-cells conveyed significantly more information than electroreceptor afferents and GFPs. We also calculated the relative reduction in SSI as a result of tail movement, at the object position at which SSI in the absence of tail movement was maximal. Results of this analysis are summarized in Figure $8 B$ (same data as in $A$ ). As expected, the relative reduction in SSI was greater for electroreceptor afferents and GFPs than for E- and I-cells.
Finally, for the subset of E- and I-cells that responded to changes in object position with clear shifts in first-spike latency as well as changes in spike number, we compared relative reduction in SSI for the two coding variables. On average, the relative reduction in SSI was smaller for spike number, although this difference did not reach statistical significance $(p=0.06 ; n=11)$.

\section{Could tail movements aid \\ electrosensory processing?}

The preceding discussion and analysis have considered changes in electrosensory input resulting from tail movements as a form of self-generated noise. Indeed, on average, the electrosensory consequences of tail movements are unlikely to convey useful information about the environment. It remains possible, however, that systematic changes in tail position, as are common during exploratory behavior, could alter the fish's electrical field in such a way as to enhance the electrical images of nearby objects. Specifically, models of electrical image generation have suggested that tail movements toward an object could increase spatial image contrast (Heligenberg, 1975; Bacher, 1983; Assad et al., 1999).

We looked for such effects in our data by comparing profiles of object-induced LEOD amplitude modulations at different tail positions. The size and shape of objectinduced modulations were similar across tail positions, differing only by an offset (supplemental Fig. $1 A, B$, available at www.jneurosci.org as supplemental material). We also recalculated SSI curves for electroreceptor afferent spike latency and GFP amplitude as a function of tail position (supplemental Fig. $1 B$, available at www.jneurosci.org as supplemental material). On average, afferent and GFP responses conveyed the same amount of information regardless of tail position (supplemental Fig. 1C, available at www.jneurosci.org as supplemental material) $(n=12)$.

Our results do not rule out the possibility that tail-probing behavior could aid electrosensory processing. Tail position-dependent increases in image contrast may depend on any number of factors, including object location relative to the electric organ, object size, distance of the object from the receptor surface, and tail angle. Notably, whereas in our experiments objects were distant from the tail/electric organ, during probing behavior fish often approach an object tail first, bringing the electric organ in close proximity to the object.

\section{Transformation of electrosensory encoding involves plastic proprioceptive inputs}

Does the reduction in the effects of tail position across processing stages involve previously described mechanisms for adaptive fil- 
A
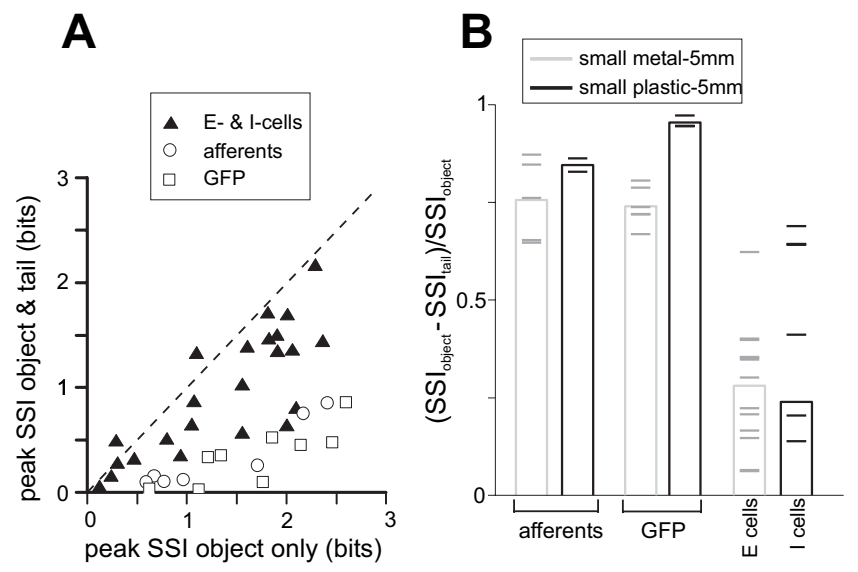

Figure 8. Summary of SSI analysis across processing stages. $\boldsymbol{A}$, Peak SSI in the presence and absence of tail movements for electroreceptor afferents, GFPs, and E-and I-cells. Analysis is with respect to first-spike latency in electroreceptor afferents and spike number in E- and I-cells. $\boldsymbol{B}$, The relative reduction in SSI (see Materials and Methods) for electroreceptor afferents, GFPs, and E- and I-cells. Analysis is with respect to first-spike latency in electroreceptor afferents and spike number in E- and I-cells. Means for each group are indicated by the height of the bars and individual experiments by horizontal lines. Smaller values indicate less information lost in the presence of tail movements. Data are the same as in $\boldsymbol{A}$.

tering? The electrosensory consequences of tail bending could be removed by anti-Hebbian synaptic plasticity at parallel fibers conveying proprioceptive information about the position of the tail. Proprioceptive fibers are a major input to granule cells that project to the ELL molecular layer via parallel fibers (Bell et al., 1992), and anti-Hebbian plasticity has been observed at parallel fiber synapses in vitro (Bell et al., 1997b). However, effects of proprioceptive inputs on E- and I-cells of the mormyrid ELL have not been reported, nor is it known whether proprioceptive input to the mormyrid ELL is plastic.

E- and I-cells often fire action potentials hundreds of milliseconds after the EOD. We noticed that in many cells, these late spikes ( $>400 \mathrm{~ms}$ after the EOD) were unaffected by the position of the object (Fig. 9B) but clearly modulated by the position of the tail (Fig. 9A). As described above, early spikes ( $<100 \mathrm{~ms}$ after the EOD) in these same cells showed a strong dependence on the object position with a substantially weaker dependence on tail position. Modulation of activity in E- or I-cells as a result of tail position could result either from the electrosensory consequences of the tail bend (presumably relayed via electroreceptor afferents) or from proprioceptive inputs conveying information about tail position (presumably relayed via parallel fibers).

To distinguish between these possibilities, we took advantage of the fact that the tail position at the time of the occurrence of a late spike was substantially different from the tail position at the time of the preceding EOD. If the late spikes were driven primarily by proprioceptive input, their occurrence should exhibit a stronger relationship to the position of the tail at the time of the spike (assuming negligible transmission time for proprioceptive information to reach ELL) than to the position of the tail at the time of the EOD. Conversely, if the late spikes were driven primarily by the electrosensory consequences of the tail bend, they should exhibit a stronger relationship to the position of the tail at the time of the EOD. For early spikes, tail position at the spike time differed from tail position at the EOD time by $<3^{\circ}$, so the two relationships were very similar (Fig. 9C,E, left). In contrast, for late spikes the two tail positions differed by $>12^{\circ}$, and it was clear in most cells that late spikes exhibited a stronger relation- ship to the position of the tail at the time of the spike (Fig. 9C,E, right).

Taking this reasoning one step further, if late spikes were actually related to tail position at the spike time and only indirectly to tail position at the EOD time, then the observed relationship with tail position at the EOD time should be unaffected by randomly reassigning tail positions at the EOD time according to their conditional distribution given the (actual) tail position at the spike time. The resulting recomputed histogram (Fig. 9C, right, gray line) agrees fairly well with the observed histogram (Fig. 9C, right, black). By comparison, the histogram resulting from reassigning tail positions at the spike time according to their conditional distribution given (actual) tail positions at the EOD time (Fig. 9E, right, gray line) agrees less well with the corresponding observed histogram (Fig. 9E, right, black). Similar results were obtained in a majority of cells and suggest that the modulation of late spikes is most closely related to the tail position at the time of the spike and thus to proprioceptive input. We also noticed that effects of tail position on early and late spikes were often opposite in sign (Fig. 9C,E, left vs right). This observation suggested a functional hypothesis: namely, that effects of tail position on late spikes reflect proprioceptive inputs acting to oppose the electrosensory consequences of the tail bend. According to this scheme, the opposite dependence of early spikes on tail position mainly reflect residual electrosensory effects of the tail bend that have not been completely cancelled by proprioceptive input. Assuming that the unopposed electrosensory consequences of an ipsilateral tail bend are excitatory in E-cells and inhibitory in I-cells, we have a qualitative prediction regarding how early and late spikes in E- and I-cells should depend on tail position.

We examined tail dependence of early and late spikes from all E- and I-cells with sufficient late spikes for analysis. Data for this analysis were from experiments in which tail and object position were simultaneously varied. The results were consistent with the functional scheme outlined above: for E-cells, early spikes were excited by an ipsilateral tail bend, whereas late spikes were inhibited (Fig. 10A) $(n=14)$. The same opposite pattern was also observed in I-cells (Fig. $10 \mathrm{~B})(n=17)$. For both E- and I-cells, late spikes were more strongly related to the tail position at the spike time, as described above (Fig. 10 A, B, right bottom vs right top). The residual tail dependence of early spikes is not inconsistent with results described earlier. Although responses of some cells were invariant to tail position, the majority showed a clear dependence on tail position that was nevertheless small relative to the dependence on object position.

To directly test the idea that proprioceptive input opposes the reafferent electrosensory consequences of tail movements, we conducted additional experiments in which we eliminated the electrosensory effects of the tail bend by shunting the EOD (see Materials and Methods). With the EOD blocked, any remaining dependence on tail position likely reflects proprioceptive input. For these experiments, only tail position was varied. We focused on I-cells, because most E-cells fired very few spikes in response to tail movements alone. Tail dependence of late spikes was similar before and after the shunt, consistent with the late spikes mainly reflecting proprioceptive input (Fig. 10D). Most importantly, shunting the EOD revealed early spikes with a strong dependence on tail position that was opposite to and, on average, larger than that observed with the EOD intact (Fig. 10C) $(n=9)$. This result provides direct support for the notion that proprioceptive inputs act to oppose the electrosensory consequences of tail movements. 
A sorted by tail position

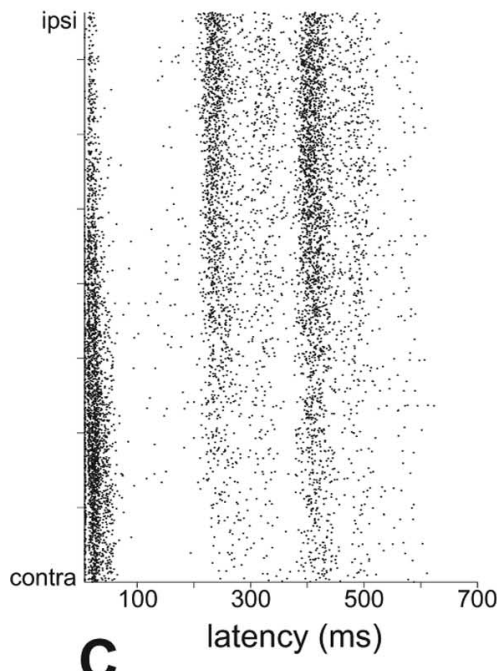

C

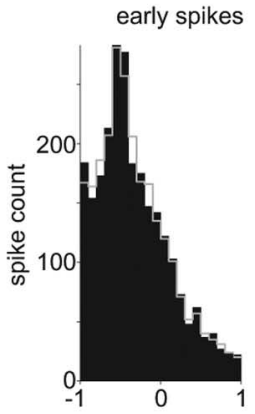

late spikes

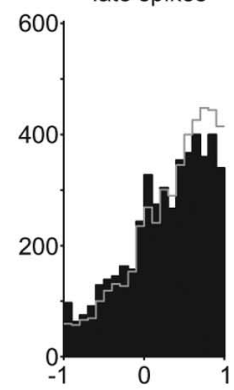

tail position at EOD time

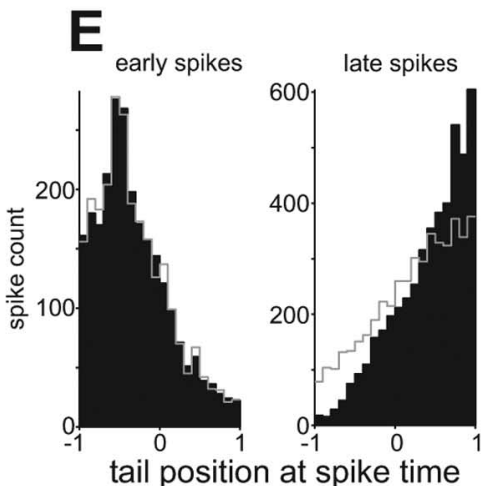

B

sorted by object position

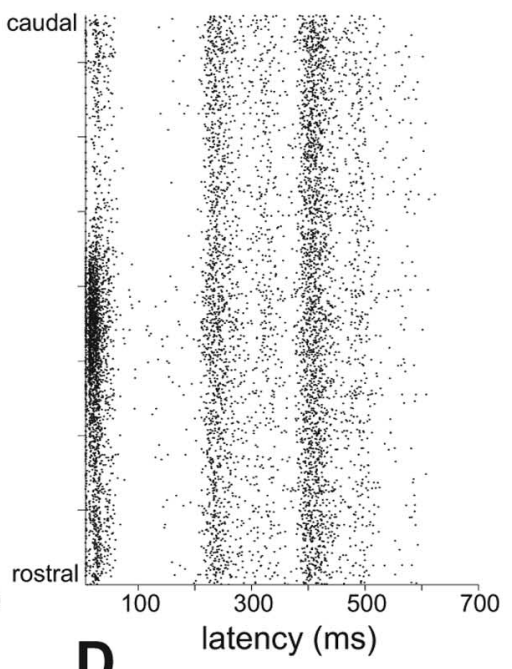

D

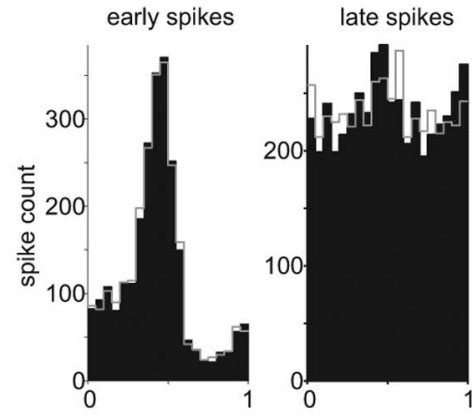

object position at EOD time

\section{$\mathbf{F}$}

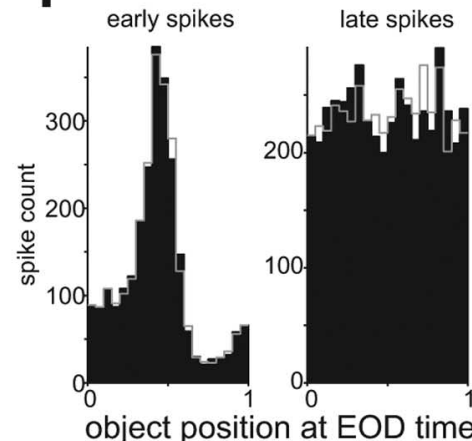

Figure 9. Dependence of late spikes on tail position in a representative I-cell. $\boldsymbol{A}, \boldsymbol{B}$, Rasters sorted by tail $(\boldsymbol{A})$ and object $(\boldsymbol{B})$ position for a representative I-cell. Note the opposite dependence of early and late spikes on tail position in $A$. $C, D$, Spike count histograms illustrating the dependence of early $(0-100 \mathrm{~ms}$; left) and late ( $>400 \mathrm{~ms}$; right) spikes on the position of the tail $(\boldsymbol{C})$ and on the position of the object $(\boldsymbol{D})$ at the time of the preceding EOD. Tail position: $-1=$ contralateral; $1=$ ipsilateral. $\boldsymbol{E}, \boldsymbol{F}$, Same data as in $\boldsymbol{C}$ and $\boldsymbol{D}$, but plotted with respect to the position of the tail/object at the time of the spike. Note that late spikes exhibit a stronger dependence on tail position at the spike time than at the EOD time (compare right panels in C and $\boldsymbol{E}$ ). See Results for explanation of predicted histograms plotted in gray in $\mathbf{C} \boldsymbol{F}$.

Anti-Hebbian plasticity at parallel fiber synapses provides a mechanism through which the effects of proprioceptive inputs could be sculpted to oppose the reafferent electrosensory consequences of tail movements. Previous studies have shown that proprioceptive input conveyed by parallel fibers contributes to the formation of negative images in the electrosensory system of elasmobranch fish (Bodznick et al., 1999) and in the gymnotid ELL (Bastian, 1995, 1996). We conducted an additional series of

experiments to determine whether proprioceptive inputs to the mormyrid ELL were plastic. Experiments were conducted in fish in which the EOD was eliminated by neuromuscular blockade, allowing us to control electrosensory stimuli and directly evaluate the influence of proprioceptive inputs on the responses of E- and I-cells. Under these conditions, many ELL neurons exhibit prominent responses to EOCD inputs associated with the spontaneously emitted motor command to discharge the electric organ. EOCD inputs are conveyed, in part, by parallel fibers, and, as demonstrated in previous studies, effects of EOCD inputs on ELL neurons are markedly plastic (Bell and Grant, 1992; Bell et al., 1997a).

We recorded EOCD-evoked responses while bending the tail. We focused mainly on I-cells, because these cells exhibited sufficient rates of EOCD-evoked spiking to reliably measure differences before and after pairing. After probing the tail dependence of EOCD-evoked responses, we paired a strong local electrosensory stimulus that inhibited cell spiking with a particular range of tail angles (one-fourth of the entire tail range, centered in the middle, near the ipsilateral extreme, or near the contralateral extreme of the range). Tail movements were the same before, during, and after the pairing period. After $15 \mathrm{~min}$ of pairing, the stimulus was turned off, and the dependence of EOCD-evoked responses on tail position was reprobed. We saw a clear increase in EOCD-evoked responses at the paired tail angle. For most cells, several pairings were conducted, each yielding an increase in spiking that was greatest at the paired tail position (Fig. $11 A, B)$. The tail dependence of EOCDevoked responses declined gradually over the course of several minutes. Plastic changes resulting from pairings at three different tail positions are summarized for seven I-cells in Figure 11C.

Qualitatively similar effects were observed in several E-cells. EOCD inputs did not reliably evoke early $(<100 \mathrm{~ms})$ spikes in E-cells. However, in some cases, pairinginduced changes were evident in late spikes occurring $>100 \mathrm{~ms}$ after the command. The nature of these changes was similar to that described above, although with the direction of changes reversed. After pairing an excitatory electrosensory stimulus with a certain range of tail positions, we observed a reduction in spiking that was greatest at the paired tail positions (data not shown). Together, these results provide clear evidence that ELL efferent cells receive plastic proprioceptive input. The changes we observed were anti-Hebbian, consistent with plasticity of proprioceptive inputs acting to oppose the electrosensory consequences of tail movements. 
It is interesting to note that before pairing in these experiments, when the EOD had been permanently silenced by neuromuscular blockade, E- and I-cell responses showed little or no dependence on tail position (Fig. 11C, top row). This observation seems to be at odds with our earlier finding that when the EOD was transiently shunted, early I-cell spikes showed a strong dependence on tail position (Fig. 10C, right) that likely reflects proprioceptive input. A possible explanation for these results is that proprioceptive negative images decay rapidly in the absence of pairing. We tested this in several experiments in which, after a period of pairing, we quickly probed tail dependence of I-cell responses and then held the tail straight for $10 \mathrm{~min}$. A final probe revealed that tail dependence had diminished significantly, even in the absence of tail bending (Fig. 11D).

Proprioceptive inputs affect efferent cells directly via excitatory parallel fiber synapses and indirectly via inhibitory interneurons. Anti-Hebbian spike timingdependent synaptic plasticity at parallel fiber synapses onto Purkinje-like interneurons, known as MG cells, has been characterized in vitro (Bell et al., 1997b; Han et al., 2000) and in vivo (Bell et al., 1993; Sawtell et al., 2007). Parallel fiber synapses onto efferent cells also exhibit anti-Hebbian plasticity (Bell et al., 1997a; van den Burg et al., 2007). As a preliminary step toward determining the site(s) of associative synaptic plasticity underlying proprioceptive negative images, we performed an additional series of experiments in which tail position was paired with postsynaptic depolarization. Assuming that the extra postsynaptic spikes induced by depolarization of a single cell are not sufficient to drive plasticity at other sites within the network, pairing-induced changes in such experiments would suggest that plasticity occurred at synapses onto the recorded cell.

We recorded EOCD-evoked synaptic responses in three LF cells while moving the tail. All three cells exhibited prominent EOCD-evoked IPSPs (Fig. 12A, B, top traces). We then paired one tail position (either the far ipsilateral or the far contralateral position) with a brief intracellular current injection that reliably evoked 1-3 spikes (Fig. 12 A, B, middle traces). After 10-15 min of pairing, we observed a substantial increase in EOCD-evoked IPSPs, with larger changes at the paired than at the unpaired angle (Fig. 12 A, B, bottom traces). We quantified these effects by measuring the area of EOCD-evoked synaptic responses at the ipsilateral and contralateral tail position, before and after pairing. EOCD-evoked IPSPs were significantly larger at the paired versus unpaired tail position in all three cells ( $p<0.01$, paired $t$ test), whereas no dependence on tail position was observed before pairing in any of the cells. Increases in IPSPs in LF cells could reflect either depression of parallel fiber excitation or potentiation of interneuron inhibition. Regardless of the precise mechanism, these results suggest that proprioceptive negative images may be
E-cells $\quad$ B $\quad$ l-cells
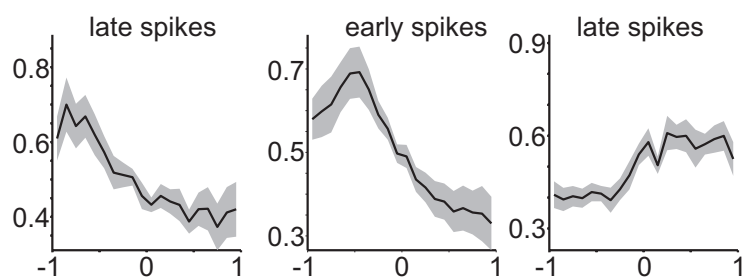

tail position at EOD time
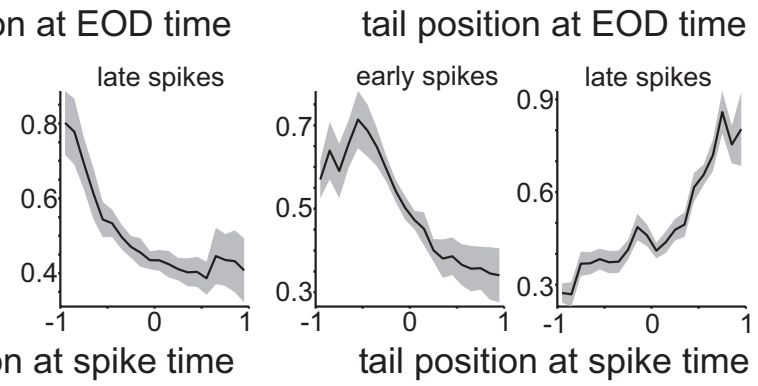

tail position at spike time

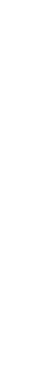
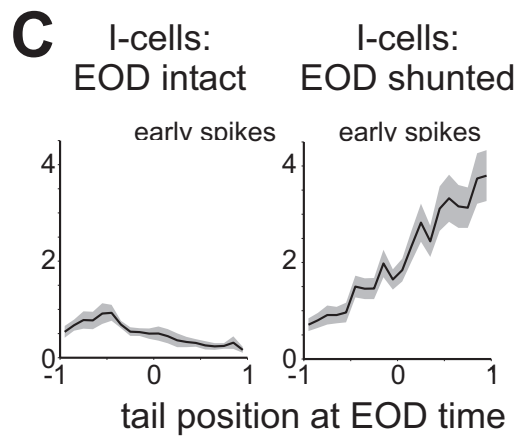

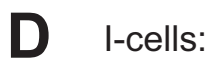
EOD intact

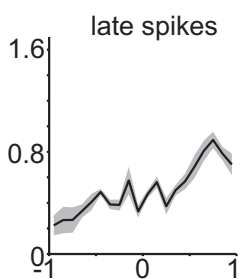

tail position at spike time
Figure 10. Evidence for proprioceptive effects that oppose the sensory consequences of tail movement. $A$, Average depencross cells. Note that the shunt reveals a dependence of early spikes on tail position that is opposite to and larger than that observed with the EOD intact. D, Shunting the EOD has little effect on late spikes in I-cells.

caused, at least in part, by plastic changes occurring at synapses onto the efferent cells themselves. Individual parallel fibers could convey EOCD information only, proprioceptive information only, or some mixture of the two. The fact that IPSPs were larger after pairing at both the paired and unpaired tail positions is consistent with efferent cells receiving a mixture of EOCD only and combined EOCD and proprioceptive parallel fibers.

\section{Discussion}

Distinguishing between self-generated sensory signals and those caused by external causes is critical for maintaining stable perception and guiding adaptive behavior (Sperry, 1950; von Holst, 1954; Cullen, 2004). Cerebellum-like structures associated with electrosensory processing provide a relatively simple model system in which to examine neural mechanisms for predicting the sensory consequences of behavior (Bell, 2001). It is hypothesized that adaptive filtering mechanisms in cerebellum-like structures improve electrosensory processing in the presence of selfgenerated signals. We provide direct evidence for such an im- 

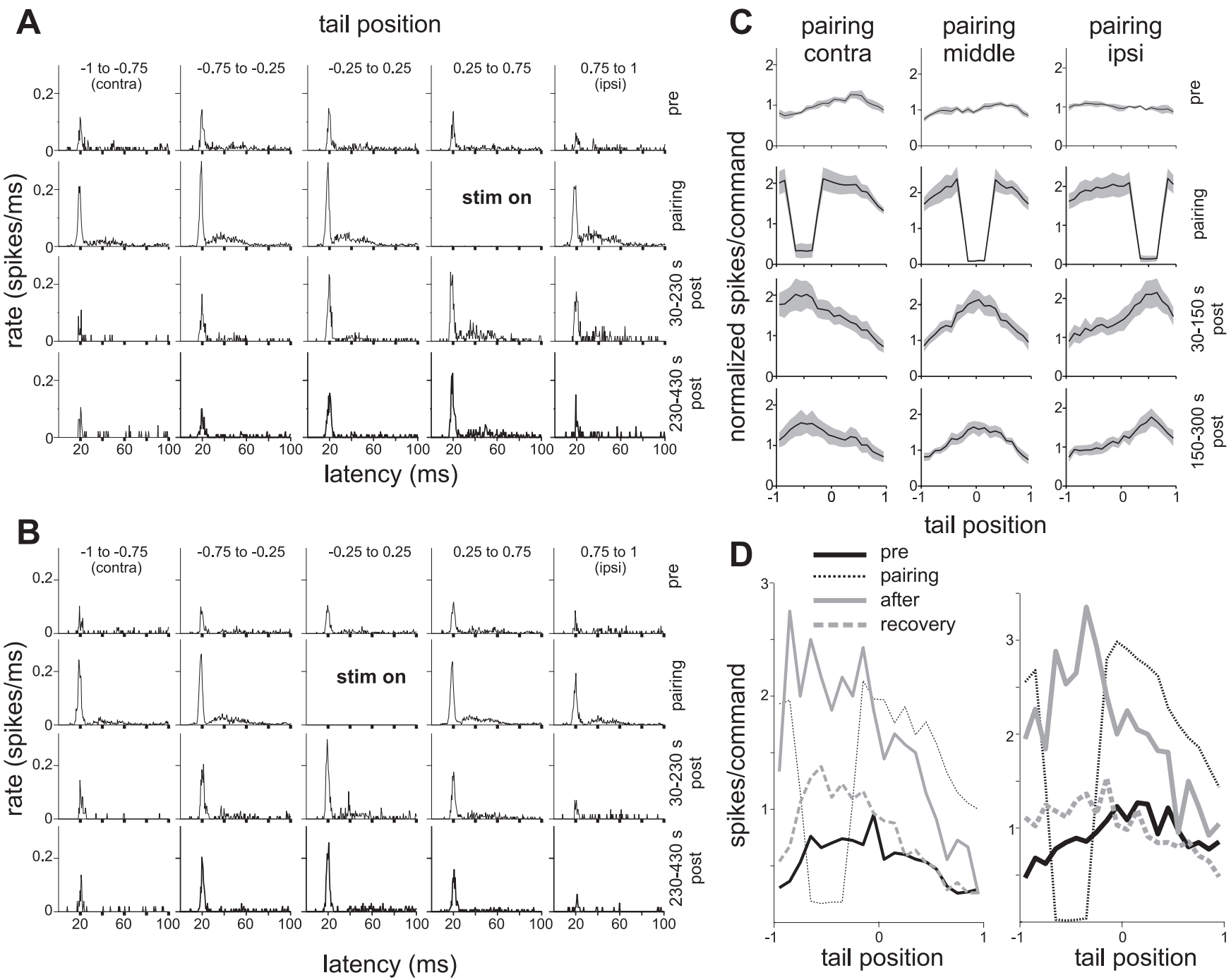

Figure 11. Plasticity of proprioceptive inputs in I-cells. A, Histograms from a representative I-cell illustrating the effects of pairing a local electrosensory stimulus with a particular range of tail positions (stim on). Columns indicate tail positions and rows different times in the experiment before, during, and after pairing. Histograms reflect electric organ corollary discharge or EOCD-evoked spiking patterns recorded under conditions in which the EOD was silenced by neuromuscular blockade. EOCD-evoked responses initially showed little dependence on tail position (top row). However, after pairing with an inhibitory stimulus (second row), EOCD-evoked responses were greatest at the position of the paired stimulus (bottom rows). $\boldsymbol{B}$, Results of a second pairing in the same cell. $\boldsymbol{C}$, Summary of pairing results from seven I-cells. For each cell, number of early spikes per EOCD was normalized by its prepairing mean across tail positions before averaging across cells. $\boldsymbol{D}$, Results from two cells of experiments probing recovery from the effects of pairing. After a brief probe to assess changes caused by pairing (solid gray curve), the tail was held straight for 10 min. A final probe (dashed gray line) indicated a substantial decay of plastic changes.

provement across processing stages. We also demonstrate that this improvement is caused, at least in part, by plastic proprioceptive inputs that oppose the electrosensory consequences of movements.

\section{Transformations of electrosensory encoding}

Our results provide the first quantitative characterization of electrosensory encoding in the mormyrid ELL. We found that individual electroreceptor afferents conveyed approximately the same amount of information about object position as individual ELL efferent cells. Whereas electroreceptor afferents encoded object position primarily via changes in spike latency, efferent cells encoded the same stimuli via changes in spike number and, in some cases, coarser changes in first-spike latency. Although we did not systematically examine other candidate codes, our results suggest a transformation from latency to rate coding. Convergence of electroreceptor afferent and precisely timed EOCD in- puts onto ELL granular cells provides a mechanism for such a recoding (Bell, 1990a; Zhang et al., 2007).

Previous studies in the electrosensory systems of elasmobranch (Bodznick et al., 1999; Montgomery and Bodznick, 1999) and weakly electric gymnotid (Bastian, 1995) fish have demonstrated that principal cells respond less strongly to self-generated signals than do electroreceptors. Our results extend this work, providing a detailed and quantitative characterization of electrosensory encoding in the presence of self-generated signals. Experiments in which object and tail position were varied simultaneously revealed that ELL efferent cell responses to objects were, in many cases, strikingly invariant to changes in tail position. Results of our information theory analysis confirm that ELL processing improves electrosensory information transmission in the presence of self-generated noise.

Although the spirit of our efforts was to simulate natural conditions, the actual challenges for active electrolocation are more 

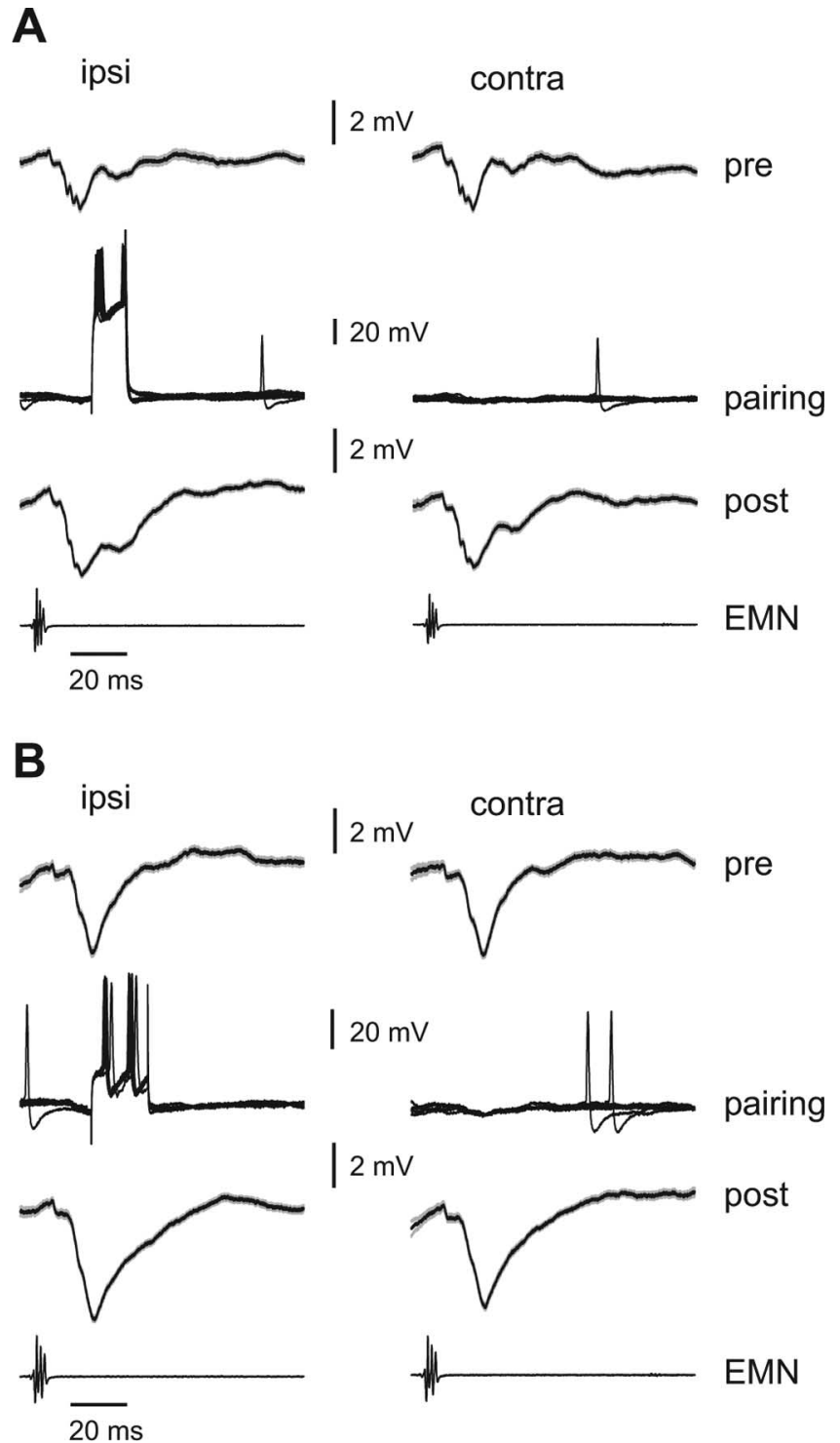

Figure 12. Effects of pairing tail position with intracellular current injections. $A, B$, IntracelIular traces from two LF cells in which cells were made to spike (via brief intracellular current injections) at a particular time after the EOD motor command ( $\sim 20 \mathrm{~ms}$ ) and at a particular position of the tail ( $30^{\circ}$ ipsilateral) (middle traces). Before pairing (pre), both LF cells exhibited an EOCD-evoked IPSP that showed no dependence on tail position (compare left and right traces in top rows in $\boldsymbol{A}$ and $\boldsymbol{B}$ ). Traces are averages of 50-60 EOCD-evoked responses. Gray outlines indicate SEM. After pairing (post), the EOCD-evoked IPSP is enhanced, and this enhancement is greater at the paired tail angle. The bottom traces, labeled EMN, are the synchronized electromotoneuron volley recorded near the tail that would normally elicit the EOD.

severe. LEOD changes resulting from invertebrate prey are likely to be substantially smaller than those produced by the objects used in our experiments (Chen et al., 2005). Moreover, the sensory consequences of movements are not fixed but depend on the nearby environment. For example, the electrosensory effects of tail bending will change from moment to moment as the fish moves from a nonconductive shelter into open water. On the other hand, under natural conditions ELL neurons may benefit from additional predictive information, e.g., descending electrosensory signals and skeletomotor corollary discharge signals. Determining the extent to which predictable sensory consequences of behavior are removed or merely reduced and on what timescales may require recordings from actively electrolocating fish.

\section{Mechanisms for minimizing self-generated signals}

The relative invariance of efferent cell responses to changes in tail position appears to be attributable, at least in part, to the effects of proprioceptive inputs conveyed by parallel fibers. Proprioceptive effects were opposite to the electrosensory effects of tail bending, consistent with proprioceptive inputs acting to reduce the sensory consequences of movements. Previously described antiHebbian plasticity at parallel fiber synapses provides a mechanism for generating proprioceptive effects that oppose reafferent sensory effects (Bell et al., 1997b; Roberts and Bell, 2000). Pairing experiments provided direct evidence that proprioceptive inputs could participate in the formation of negative images. Intracellular recordings from efferent cells in which tail position was paired with depolarization suggest that proprioceptive inputs to the efferent cells themselves are sites of plastic change. This result is consistent with a simple scheme in which predictable sensory consequences of behavior on ELL output are opposed directly by plastic changes in parallel fiber synapses onto the efferent cells themselves. Similar plasticity of parallel fiber inputs has been reported in elasmobranch (Bodznick et al., 1999) and gymnotid (Bastian, 1995, 1996) fish. The present results further underscore the remarkable similarities in adaptive processing mechanisms across taxa (Bell, 2001).

The rapid decay of the plastic changes we observed, even in the absence of tail bending, suggests that proprioceptive negative images must be actively maintained and explains the lack of dependence on tail position under conditions in which the EOD is permanently blocked. This rapid decay contrasts with the relative stability of negative images generated by EOCD inputs (Bell, 1986). The difference may lie not in the plasticity mechanisms themselves, but in the nature of presynaptic activity patterns. Unlike EOCD inputs, proprioceptive mossy fiber inputs fire tonically. If parallel fibers conveying proprioceptive input also have some tonic activity, ongoing nonassociative potentiation (Bell et al., 1997b; Roberts and Bell, 2000) might be expected to erase previous learning under conditions in which tail position is uncoupled from reafference. The observed modulation of efferent cell late spikes by tail position is consistent with tonic proprioceptive inputs.

Several key questions remain regarding the implementation of adaptive filtering at the level of ELL circuitry. In addition to direct effects of excitatory parallel fiber synapses onto efferent cell apical dendrites, proprioceptive inputs likely affect efferent cells via inhibition mediated both by stellate cells and Purkinje-like MG cells. In fact, the majority of parallel fiber synapses in the ELL are onto the spine-covered apical dendrites of MG cells. These synapses are also the site of prominent anti-Hebbian spike timingdependent synaptic plasticity (Bell et al., 1997b; Sawtell et al., 2007). MG cells inhibit nearby efferent cells, an arrangement similar to that found in the teleost cerebellum, where Purkinje cells terminate locally on cerebellar output neurons, and similar also to the mammalian dorsal cochlear nucleus, where Purkinjelike cartwheel cells inhibit nearby efferent cells (Bell, 2002). How anti-Hebbian plasticity at parallel fiber-MG cell synapses contributes to adaptive filtering is an important question for future studies.

Several lines of evidence suggest that inhibitory synapses in ELL may also be plastic. It will be of interest to determine whether the larger IPSPs we observed after pairing in LF cells (Fig. 12) are attributable to depression of parallel fiber excitation or to potentiation of inhibitory (MG-efferent cell or stellate cell-efferent) synapses.

In addition to adaptive mechanisms described above, spatial 
filtering mediated by opponent center-surround receptive fields may also contribute to minimizing effects of tail bending. In our experiments, the spatial profiles of LEOD amplitude modulation caused by changes in tail position were significantly wider than those caused by changes in object position. Although we cannot directly judge the relative importance of spatial filtering versus adaptive mechanism, the strong modulation of efferent cell responses by tail position evident after shunting the EOD is consistent with an important role for proprioceptive inputs. The electrosensory system has no functional equivalent of a lens; thus, the width of electrical images depends strongly on the size and distance of objects. Electrical images cast by large, distant objects may be effectively uniform over the width of efferent cell RFs. Adaptive mechanisms might be especially critical under such conditions.

\section{Implications for cerebellar function}

The generation of sensory predictions in cerebellum-like structures described here is similar to the hypothesized role of the mammalian cerebellum in learning to predict the sensory consequences of motor commands (Miall et al., 1993; Wolpert et al., 1998). Such predictions, known as forward models, are likely critical for rapid and flexible control of movements (Bastian, 2006). Studies of adaptive processing in cerebellum-like structures may thus be relevant for understanding the role of the cerebellum itself in learning and prediction. For example, forward model predictions about the sensory consequence of a motor command must take present context into account; e.g., the sensory consequence of a motor command to initiate a reach will depend on the starting position of the limb. The present work provides, at the level of single-neuron responses, a simple demonstration of predictive changes in the effects of a motor command (the electric organ corollary discharge) that depend on proprioceptive context (tail position).

Studies of adaptive processing in cerebellum-like structures may also be relevant for understanding mechanisms of cerebellar learning. The depression of responses to signals conveyed by parallel fibers after the pairing of these signals with postsynaptic excitation in cerebellum-like structures is similar to the depression of responses to parallel fiber stimulation in the mammalian Purkinje cells after pairing with climbing fiber input (Ito, 2001). Such depression has been linked to the formation of negative images of predicted sensory input in cerebellum-like structures and to motor learning in the mammalian cerebellum (Ito, 1984). It is of interest in this regard that patterns of stimulus-driven parallel fiber-evoked simple spike activity in Purkinje cells are often close to the inverse of climbing fiber responses. For example, cutaneous parallel fiber receptive fields in the C3 zone of the cerebellum are outside of and, seemingly, opposite to climbing fiber receptive fields (Ekerot and Jörntell, 2003). Thus, simple spike activity may be seen as a kind of negative image of predicted climbing fiber activity. Plasticity at parallel fiber synapses may play a role in generating this relationship (Jörntell and Ekerot, 2002).

\section{References}

Assad C, Rasnow B, Stoddard PK (1999) Electric organ discharges and electric images during electrolocation. J Exp Biol 202:1185-1193.

Bacher M (1983) A new method for the simulation of electric fields, generated by electric fish and their distortions by objects. Biol Cybern 47:51-58.

Bastian AJ (2006) Learning to predict the future: the cerebellum adapts feedforward movement control. Curr Opin Neurobiol 16:645-649.

Bastian J (1995) Pyramidal-cell plasticity in weakly electric fish: a mecha- nism for attenuating responses to reafferent electrosensory inputs. J Comp Physiol 176:63-78.

Bastian J (1996) Plasticity in an electrosensory system. I. General features of dynamic sensory filter. J Neurophysiol 76:2483-2496.

Bell CC (1981) An efference copy modified by reafferent input. Science 214:450-453.

Bell CC (1986) Duration of plastic change in a modifiable efference copy. Brain Res 369:29-36.

Bell CC (1990a) Mormyromast electroreceptor organs and their afferents in mormyrid electric fish: II. Intra-axonal recordings show initial stages of central processing. J Neurophysiol 63:303-318.

Bell CC (1990b) Mormyromast electroreceptor organs and their afferents in mormyrid electric fish: III. Physiological differences between two morphological types of fibers. J Neurophysiol 63:319-332.

Bell CC (2001) Memory-based expectations in electrosensory systems. Curr Opin Neurobiol 11:481-487.

Bell CC (2002) Evolution of cerebellum-like structures. Brain Behav Evol 59:312-326.

Bell CC, Grant K (1992) Corollary discharge effects and sensory processing in the mormyromast regions of the mormyrid electrosensory lobe: II. Cell types and corollary discharge plasticity. J Neurophysiol 68:859-875.

Bell CC, Grant K, Serrier J (1992) Corollary discharge effects and sensory processing in the mormyrid electrosensory lobe: I. Field potentials and cellular activity in associated structures. J Neurophysiol 68:843-858.

Bell CC, Caputi A, Grant K, Serrier J (1993) Storage of a sensory pattern by anti-Hebbian synaptic plasticity in an electric fish. Proc Natl Acad Sci USA 90:4650-4654.

Bell CC, Caputi A, Grant K (1997a) Physiology and plasticity of morphologically identified cells in the mormyrid electrosensory lobe. J Neurosci 17:6409-6422.

Bell CC, Han VZ, Sugawara S, Grant K (1997b) Synaptic plasticity in a cerebellum-like structure depends on temporal order. Nature $387: 278-281$.

Bodznick D, Montgomery JC, Carey M (1999) Adaptive mechanisms in the elasmobranch hindbrain. J Exp Biol 202:1357-1364.

Butts DA (2003) How much information is associated with a particular stimulus? Network 14:177-187.

Butts DA, Goldman MS (2006) Tuning curves, neuronal variability, and sensory coding. PLoS Biol 4:e92.

Chen L, House JL, Krahe R, Nelson ME (2005) Modeling signal and background components of electrosensory scenes. J Comp Physiol 191:331-345.

Cullen KE (2004) Sensory signals during active versus passive movement. Curr Opin Neurobiol 14:698-706.

DeWeese MR, Meister M (1999) How to measure the information gained from one symbol. Network 10:325-340.

Ekerot CF, Jörntell H (2003) Parallel fiber receptive fields: a key to understanding cerebellar operation and learning. Cerebellum 2:101-109.

Engelmann J, Bacelo J, van den Burg E, Grant K (2006) Sensory and motor effects of etomidate anesthesia. J Neurophysiol 95:1231-1243.

Gomez L, Budelli R, Grant K, Caputi AA (2004) Pre-receptor profile of sensory images and primary afferent neuronal representation in the mormyrid electrosensory system. J Exp Biol 207:2443-2453.

Han VZ, Grant G, Bell CC (2000) Reversible associative depression and nonassociative potentiation at a parallel fiber synapse. Neuron 27:611-622.

Heligenberg W (1975) Theoretical and experimental approaches to spatial aspects of electrolocation. J Comp Physiol 103:247-272.

Ito M (1984) The cerebellum and neural control. New York: Raven.

Ito M (2001) Cerebellar long-term depression: characterization, signal transduction, and functional roles. Physiol Rev 81:1143-1195.

Jörntell H, Ekerot CF (2002) Reciprocal bi-directional plasticity of parallel fiber receptive fields in cerebellar Purkinje cells and their afferent interneurons. Neuron 34:797-806.

Meek J, Grant K, Bell C (1999) Structural organization of the mormyrid electrosensory lateral line lobe. J Exp Biol 202:1291-1300.

Miall RC, Weir DJ, Wolpert DM, Stein JF (1993) Is the cerebellum a Smith Predictor? J Motor Behav 25:203-216.

Migliaro A, Caputi AA, Budelli R (2005) Theoretical analysis of prereceptor image conditioning in weakly electric fish. PLoS Comput Biol $1: 123-131$.

Miller G (1955) Note on bias of information estimates. In: Information the- 
ory in psychology: problems and methods II-B (Quastler H, ed), pp 95100. Glencoe, IL: Free.

Mohr C, Roberts PD, Bell CC (2003) Cells of the mormyromast region of the mormyrid electrosensory lobe: I. Responses to the electric organ corollary discharge and to electrosensory stimuli. J Neurophysiol 90:1193-1210.

Montgomery JC, Bodznick D (1999) Signals and noise in the elasmobranch electrosensory system. J Exp Biol 202:1349-1355.

Roberts PD, Bell CC (2000) Computational consequences of temporally asymmetric learning rules: II. Sensory image cancellation. J Comput Neurosci 9:67-83.

Sawtell NB, Mohr C, Bell CC (2005) Recurrent feedback in the mormyrid electrosensory system: cells of the preeminential and lateral toral nuclei. J Neurophysiol 93:2090-2103.

Sawtell NB, Williams A, Bell CC (2007) Central control of dendritic spikes shapes the responses of Purkinje-like cells through spike timingdependent synaptic plasticity. J Neurosci 27:1552-1565.
Sawtell NB, Williams A, Roberts PD, von der Emde G (2006) Effects of sensing behavior on a latency code. J Neurosci 26:8221-8234.

Sperry RW (1950) Neural basis of the spontaneous optokinetic response produced by visual inversion. J Comp Psychol 42:482-489.

van den Burg EH, Engelmann J, Bacelo J, Gomez L, Grant K (2007) Etomidate reduces initiation of backpropagating dendritic action potentials: implications for sensory processing and synaptic plasticity during anesthesia. J Neurophysiol 97:2373-2384.

von Holst E (1954) Relations between the central nervous system and the peripheral organs. Br J Animal Behav 2:89-94.

Wolpert DM, Miall C, Kawato M (1998) Internal models in the cerebellum. Trends Cogn Sci 2:338-347.

Zhang J, Han VZ, Meek J, Bell CC (2007) Granular cells of the mormyrid electrosensory lobe and postsynaptic control over presynaptic spike occurrence and amplitude through an electrical synapse. J Neurophysiol 97:2191-2203. 